deprived target. Our results support the intuitive idea that acute isolation causes social craving, similar to the way fasting causes hunger.

\section{Reporting summary.}

Further information on research design is available in the Nature Research Reporting Summary linked to this article.

How are people affected by a period of forced social isolation? Chronic social isolation and loneliness are associated with lower physical ${ }^{1,2}$ and mental ${ }^{2-4}$ health, but little is known about the consequences of acute mandatory isolation. Positive social interactions in and of themselves may be basic human needs, analogous to other basic needs like food consumption or sleep 5,6 . If so, the absence of positive social interaction may create a want, or 'craving', that motivates behavior to repair what is lacking'. Cues associated with positive social interaction (for example, smiling faces) activate neural reward systems ${ }^{7}$. However, research on the neural representation of unmet human social needs is scarce ${ }^{8}$.

In social animals, social interactions act as primary rewards ${ }^{9-11}$ : they are inherently pleasurable and motivate behavior in the absence of any other reward. Extended periods of isolation, especially during development, can dramatically disrupt behavior and brain function ${ }^{12-14}$. Even a brief acute period of social isolation causes an aversive, 'lonelinesslike' brain state in adult mice, causing the mice to seek social interaction ${ }^{15}$ which is mediated specifically by dopaminergic (DA) midbrain neurons ${ }^{16}$, similar to other kinds of craving ${ }^{17}$.

However, the similarity to human loneliness has been $\operatorname{disputed}^{8}$, and it is not possible to assess whether a mouse subjectively feels lonely when isolated. Would acute isolation evoke a similar response in humans? The intuitive idea that depriving social needs evokes social craving, analogous to the way fasting evokes food craving and is mediated by similar DA midbrain regions, has never been directly tested in humans. The few previous studies investigating the correlation between self-reported chronic loneliness and brain responses to social stimuli yield contradictory findings ${ }^{18-20}$, and are limited by the ambiguity of observed correlations: if brain responses do differ, are the differences antecedents or effects of loneliness?

\section{Results}

To address these questions, we experimentally induced social isolation in a within-subject design; 40 healthy, socially connected young adults (ages 18-40, 27 women) underwent $10 \mathrm{~h}$ of social isolation and functional magnetic resonance imaging (fMRI) with a cueinduced craving (CIC) paradigm. Each participant also underwent $10 \mathrm{~h}$ of food fasting and subsequent MRI. Figure 1 shows an overview of the experimental procedures (see Methods for details). All predictions and methods were preregistered on the Open Science Framework (OSF; https://osf.io/cwg9e).

All participants were within a healthy weight range (body mass index (BMI) mean (s.d.) $=22.8(2.2)$ ), reported frequent social interactions (monthly interactions, mean $=49.1$ 
(31.7); minimum $=10)$ and close relationships (number of close relationships, mean = 12.3 (5.1); minimum = 3). Participants reported relatively ${ }^{21}$ low levels of pre-existing loneliness (University of California, Los Angeles (UCLA) loneliness scale, mean = 33.2 (6.3), maximum $=47$ out of 80$)$.

\section{Subjective social craving can be evoked by acute objective social isolation.}

Could we experimentally induce the subjective experience of social isolation in human participants? Human loneliness is not a simple product of objective isolation: people can be alone without feeling lonely or feel lonely even in a crowd $\mathrm{d}^{22}$. Moreover, experimentally induced isolation would be brief, relative to the human lifespan, and, for ethical reasons, human participants (unlike rodents) would be able to predict when the isolation would end. In all, the first challenge of this research was to develop an experimental induction of objective isolation that created the subjective experience of unmet social needs in human participants. To address this challenge, we had socially connected healthy human adults spend $10 \mathrm{~h}$ (9:00 to 19:00) alone, with no social interaction and no other social stimulation (for example, social media, email, fiction). We used self-report questionnaires to assess people's resulting subjective experience of loneliness and social craving.

After $10 \mathrm{~h}$ of social isolation, participants reported substantially increased social craving (Student's $t$-test, $t(39)=5.00, P<0.001)$, loneliness $(t(39)=5.17, P<0.001)$, discomfort $(t(39)=5.57, P<0.001)$ and dislike of isolation $(t(39)=4.13, P<0.001)$, and decreased happiness $(t(39)=-4.21, P<0.001)$ compared with when they started isolation. Of the 40 individual participants, 36 reported feeling more lonely after isolation. As expected, after $10 \mathrm{~h}$ of food fasting, participants reported increased food craving $(t(36)=17.40, P<$ $0.001)$, hunger $(t(36)=23.90, P<0.001)$, discomfort $(t(36)=13.56, P<0.001)$ and dislike of fasting $(t(36)=6.28, P<0.001)$, and decreased happiness $(t(36)=-3.05, P=0.004)$ compared with when they started fasting. Thus, both forms of abstinence evoked craving for the specifically deprived need, along with general discomfort and decreased happiness. However, we note that social craving after isolation was more variable across participants than food craving after fasting (mean(s.d.) of craving ratings, after $10 \mathrm{~h}$ : food craving $=80.25(19.39)$; social craving $=66.38(24.52)$, Fig. 2 ; Levene's test indicated unequal variances $(F(76)=15.86, P<0.001))$.

\section{After isolation, social cues evoke neural signatures of craving.}

In primates, aversive motivation (that is, a negative state such as hunger or pain that motivates behavior to relieve the state ${ }^{23}$ ) is represented in the substantia nigra pars compacta and ventral tegmental area $\left(\mathrm{SN} / \mathrm{VTA}^{24}\right)$, and the SN/VTA is activated by craving for food and for drugs of addiction ${ }^{25-27}$. In the $\mathrm{SN}$, in particular, $70 \%$ of the neurons are DA, so fMRI signals in this brain area probably mainly reflect DA neuron activity ${ }^{28}$. We therefore hypothesized that acute isolation in humans might produce a social craving response in the SN/VTA. Neuroimaging of the SN/VTA poses a technical challenge, however, because it is a small structure, adjacent to the sphenoid sinus (a large, air-filled cavity located anterior to the brainstem), and is therefore prone to distortions and signal $\operatorname{loss}^{28}$. To address this challenge, we optimized MR image acquisition parameters, and used a newly developed atlas (see Methods for details) to identify the SN/VTA in individual participants' brains. We 
also included an independent functional localizer task to identify voxels in each participant's midbrain that are maximally sensitive to expected reward and novelty, consistent with DA activity ${ }^{29}$.

To measure DA midbrain responses to food cues and social cues, we developed a CIC paradigm, in which participants viewed pictures of their favorite social activities, favorite foods and a pleasant control (flowers). We included favorite foods so that we could compare social craving, within participants, to a well-established neural craving response, evoked by viewing food cues after several hours of fasting ${ }^{26}$. In the anatomically defined SN/VTA (Fig. 3a), responses to food cues were higher after fasting than after isolation (beta $(b)=$ $0.06, t=3.1,95 \%$ confidence interval $(\mathrm{CI})=(0.0 .02,0.09), P=0.002)$, but responses to social cues were not significantly higher after isolation than after fasting $(b=0.006, t=0.7$, $95 \% \mathrm{CI}=(-0.01,0.03), P=0.50$; for full results of the model including all main effects, see Supplementary Table 1). In the midbrain functional region of interest (ROI) (voxels maximally sensitive to reward and novelty; Fig. $3 b$ ), responses to food cues were higher after fasting than after isolation $(b=0.03, t=3.3,95 \% \mathrm{CI}=(0.01,0.05), P=0.001)$, and responses to social cues were higher after isolation than after fasting ( $b=0.03, t=2.5,95 \%$ $\mathrm{CI}=(0.006,0.05), P=0.01$; see Supplementary Table 2 for full results $)$.

We also compared SN/VTA responses with food and social cues after fasting and isolation to responses to the same cue on the baseline day (when participants' food intake and social interactions were not controlled or measured). Unexpectedly, when compared with the baseline day, deprivation led to a decreased response to the nondeprived cue, rather than an enhanced response to the deprived cue (Fig. 3 and Supplementary Fig. 2). In the anatomically defined SN/VTA, responses to food cues were numerically but not significantly higher after fasting than baseline, but significantly lower after isolation than baseline ( $b$ $=-0.05, t=-2.1,95 \% \mathrm{CI}=(-0.11,-0.003), P=0.038)$; responses to social cues $\mathrm{did}$ not significantly differ from responses at baseline for any of the sessions. In the midbrain functional ROI, responses to food cues were numerically but not significantly higher after fasting and significantly lower after isolation than baseline $(b=-0.06, t=-2.4,95 \% \mathrm{CI}=$ $(-0.12,-0.01), P=0.02)$, whereas social cues were numerically but not significantly higher after isolation than baseline and showed a trend to be lower after fasting ( $b=0.01, t=-1.8$, $95 \% \mathrm{CI}=(-0.11,-0.004), P=0.07$; see Supplementary Tables 3 and 4 for full results $)$

SN/VTA activity was correlated with self-reported craving, for both food and social cues. However, this correlation was significant for different measures in the two conditions. We measured self-reported craving in two ways: craving ratings during the task directly in response to the craving cues (Food_Craving_CICISocial_Craving_CIC) and craving ratings on the questionnaire before participants went into the scanner (Food_Craving_Q/ Social_Craving_Q). The two craving measures (Craving_CIC and Craving_Q) were correlated across participants in both conditions (food, Pearson's $r$ : $r(36)=0.52 ; P<0.001$; social: $r(38)=0.30 ; P=0.030$; see Methods for details). We consider the two self-report measures to be estimates of the same hypothesized psychological process, and report results as significant after Bonferroni's correction for the two tests. After fasting, in the anatomical SN/VTA, the response to food cues (versus flowers) was positively correlated with the participant's self-reported food craving measured during the CIC task (Food_Craving_CIC. 
$r(38)=0.31 ; P=0.025$; Fig. $3 \mathrm{c})$, but not with food craving on the final questionnaire (Food_Craving_Q,$r(36)=0.05 ; P=0.375)$. As the data for food craving measured during the CIC task is truncated at the upper limit of 10 , we also analyzed the correlation using a truncated regression model (see Methods for details): SN/VTA activity in response to food cues remained associated with higher craving ratings during the task (Food_Craving_CIC: $b=0.04, t=2.10, P=0.038$ ). After isolation, the SN/VTA response to social cues (versus flowers) was positively correlated with self-reported social craving on the final questionnaire (Social_Craving_ $Q, r(38)=0.36 ; P=0.011$; Fig. $3 c$ ), but not with social craving measured during the CIC task (Social_Craving_CIC. $r(38)=0.054 ; P=0.370)$.

As a direct test of the similarity of activity patterns for food cues after fasting, and social cues after isolation, we implemented a multivoxel pattern analysis. We trained a classifier on the pattern of activity in the SN/VTA after fasting to food cues and flower images. This trained classifier then generalized to above-chance decoding ( $a=0.001$; see Methods for details and Fig. 4 for an illustration of our multivariate analysis approach) of social cues from flower images after isolation (mean accuracy $=0.542$, bootstrapped $\mathrm{CI}=(0.505,0.581)$, $P<0.001$ ), but did not show significant above-chance accuracy at baseline (mean accuracy $=0.534$, bootstrapped $\mathrm{CI}=(0.497,0.573), P=0.007)$. However, it is important to note that the classification accuracy for social cues did not differ between the isolation and baseline sessions.

In addition, the classifier was able to decode food from flower images after isolation and baseline (isolation: mean accuracy $=0.540$, bootstrapped CI $=(0.494,0.584), P<0.001$; baseline: mean accuracy $=0.545$, bootstrapped $\mathrm{CI}=(0.509,0.594), P<0.001)$. Given that the classification algorithm was always used to distinguish between a target stimulus and flowers, one might worry that the generalization is simply due to learning about flowers. We addressed this concern using a representational similarity analysis that directly compared food and social cues in deprived or nondeprived states.

The pattern of SN/VTA activity in response to social cues on the isolation day was more similar to the pattern of food cues on the fasting day than to food cues on the baseline day (mean Fisher-transformed dissimilarity: social_craved-food_craved $=0.87$, social_craved-food_noncraved $=1.09 ; t(39)=2.0, P=0.025)$. Indeed, SN/VTA responses to different stimuli in a similar motivational state (social_craved-food_craved) trended toward being more similar to each other than the responses to the same stimuli across different motivational states (food_craved-food_noncraved; mean correlation $=1.03 ; t(39)=1.4, P=$ 0.08).

Correlations with chronic loneliness.-In summary, these results suggest that, across all participants, the SN/VTA shows an increased response to social cues after objective social isolation compared with after fasting, with a spatial pattern that is similar to the response to food cues when hungry. The magnitude of this response was variable across participants, and larger in those who reported more social craving after the acute isolation period. We predicted that individual variability in response to objective isolation might reflect pre-existing differences in participants' social network size and/or chronic loneliness. Consistent with this prediction, participants with higher levels of chronic loneliness during 
the initial screening reported less craving for social contact in response to the social cues in the CIC task (Social_Craving_CIC. $r(38)=-0.37 ; P=0.020$ ), and somewhat less craving after $10 \mathrm{~h}$ of isolation on the online questionnaire (Social_Craving_Q: $r(38)=-0.30 ; P=$ 0.059). People with higher pre-existing chronic loneliness also showed a muted response in SN/VTA to social cues after acute isolation $(r(38)=-0.33 ; P=0.036)$. Individual differences in social network size did not predict either self-reported or neural responses to acute social isolation (all $P$ values $>0.33$ ). We explored whether pre-existing loneliness was associated with different responses to food cues after fasting and found that, although loneliness did not affect self-reported food craving $(P=0.431)$, higher loneliness was associated with a trend toward lower post-fasting SN/VTA responses to food cues $(r(38)=$ $-0.30 ; P=0.062)$.

SN/VTA: correlations with cravings.-In our preregistration, we planned to test whether the magnitude of the SN/VTA response to a specific deprived cue (food or social) was correlated with self-reported craving for that cue. As reported in After isolation, social cues evoke neural signatures of craving, these predicted correlations were observed. In addition to these preregistered analyses, as prompted by a reviewer, we explored the broader structure of correlations between behavioral and neural measures of craving.

We tested whether the correlations between the SN/VTA and self-reported craving (on the CIC task) were restricted to the deprived day. We found that they were not. Self-reported craving for food was correlated with the SN/VTA response to food cues on both nonfasted sessions (baseline session: $r(38)=0.32 ; P=0.045$; isolation session: $r(38)=0.43, P=$ 0.006). As the data for food craving measured during the CIC task were truncated at the upper limit of 10, we also analyzed the correlations using a truncated regression model (see Methods for details): for both nonfasted sessions, SN/VTA activity in response to food cues remained associated with higher craving ratings during the task (baseline: $b=0.02$, $t=2.11, P=0.034$; isolation: $b=0.02, t=3.00, P=0.003$ ). Self-reported craving for social interaction was correlated with the SN/VTA response to social cues on both the nonisolated sessions (baseline session: $r(38)=0.37 ; P=0.018$; fasting session: $r(38)=0.35$, $P=0.027)$. Thus, individual differences in the magnitude of the SN/VTA response to a cue are correlated with simultaneously measured self-reported craving for that cue, whether or not that cue has been selectively deprived.

\section{Mixed-effects model: striatum.}

Although our primary hypotheses focused on the DA midbrain, and particularly the SN/ VTA, we also investigated responses in the striatum, a major target of projections from midbrain DA neurons ${ }^{30}$. A mixed-effects regression model tested effects of cue (food, social, flowers) and session (fasting, isolation) for each subregion of the striatum separately (nucleus accumbens (NAcc), caudate nucleus $(\mathrm{Ca})$ and putamen $(\mathrm{Pu})$ ). We report results as significant at $P<0.017(0.05 / 3)$.

Food cues (compared with flowers) evoked a stronger response after fasting (compared with after isolation) in NAcc $(b=0.08, t=4.7,95 \% \mathrm{CI}=(0.05,0.11), P<0.001 ;$ Fig. 5$)$ and $\mathrm{Pu}(b=0.03, t=3.2,95 \% \mathrm{CI}=(0.01,0.05), P=0.002)$, but not in $\mathrm{Ca}(b=0.025, t=1.9$, 
95\% CI $=(-0.001,0.05), P=0.061)$. Social cues evoked a stronger response after isolation (compared with after fasting) in $\mathrm{Ca}(b=0.04, t=3.0,95 \% \mathrm{CI}=(0.01,0.06), P=0.003)$ but not in NAcc $(b=0.04, t=2.1,95 \% \mathrm{CI}=(0.002,0.07), P=0.04)$ and $\mathrm{Pu}(b=0.02$, $t=1.4,95 \% \mathrm{CI}=(-0.007,0.04), P=0.17)$. Similar to the SN/VTA, when compared with the baseline day, deprivation led to a decreased response to the nondeprived cue in striatal subregions, rather than an enhanced response to the deprived cue (an enhanced response to social cues after isolation compared with baseline in the caudate did not survive correction for multiple comparisons). For full results, see Supplementary Tables 5-12.

Thus, although the responses in SN/VTA were similar and overlapping for food craving and social craving, responses in the striatum were dissociable. We conducted exploratory analyses to test whether subjective craving of food and social contact (Craving_CIC) was correlated with striatal activity for any condition or any of the three subregions (reporting results significant at $a<0.017$ to correct for multiple comparisons) and found no significant correlation in the striatum ROIs (all $P$ values $>0.038$ ).

\section{Exploratory analyses: downstream targets of the SN/VTA.}

We also conducted exploratory analyses testing the effects of food and social craving in other brain regions associated with craving. We selected four ROIs based on meta-analyses of craving across different modalities (see Methods for details): amygdala, insular cortex, anterior cingulate cortex (ACC) and orbitofrontal cortex (OFC). A mixed-effects regression model tested effects of cue (food, social, flowers) and session (fasting, isolation) for each region and we reported results as significant at $P<0.0125(0.05 / 4)$, to account for the four regions tested.

Food cues (compared with flowers) evoked a stronger response after fasting (compared with after isolation) in the ACC $(b=0.07, t=2.9,95 \% \mathrm{CI}=(0.02,0.12), P=0.005)$ and trending stronger responses after fasting in the insula $(b=0.03, t=2.0,95 \% \mathrm{CI}=(0.0003,0.07), P=$ $0.052)$ and amygdala $(b=0.04, t=1.9,95 \% \mathrm{CI}=(0.0007,0.07), P=0.055)$, but not in the OFC $(b=0.03, t=1.0,95 \% \mathrm{CI}=(-0.1,0.04), P=0.337)$.

Social cues evoked a stronger response after isolation (compared with after fasting) in the OFC $(b=0.11, t=2.53,95 \% \mathrm{CI}=(0.03,0.20), P=0.012)$, but in none of the other ROIs (ACC: $b=-0.007, t=-0.3,95 \% \mathrm{CI}=(-0.06,0.05), P=0.787$; insula: $b=0.02, t=1.0$, $95 \% \mathrm{CI}=(-0.06,0.02), P=0.324$; amygdala: $b=0.02, t=1.0,95 \% \mathrm{CI}=(-0.02,0.06)$, $P=0.334)$. When compared with the baseline day, we found no significant effects in the exploratory ROIs. For full results, see Supplementary Tables 13-20.

An exploratory whole-brain, random-effects analysis yielded converging results: selective responses to food craving in the NAcc, ACC, periaqueductal gray and amygdala, and selective responses to social craving in the $\mathrm{Ca}, \mathrm{OFC}$ and dorsomedial prefrontal cortex (Supplementary Tables 21 and 22 and Supplementary Fig. 4). A conjunction analysis assessing overlapping activation between the food contrast (fasting > isolation) and the social contrast (isolation > fasting) did not show any suprathreshold voxels. 
We also calculated exploratory whole-brain contrasts against baseline (that is, food: fasting $>$ baseline and social: isolation > baseline) but found no suprathreshold activity in this analysis. This result is in line with our ROI analysis in the SN/VTA showing similar magnitude of activity in in response to food cues after fasting and baseline, and in response to social cues after isolation and baseline.

\section{Exploratory behavioral analyses.}

Correlations between craving ratings.-We explored whether individual differences in self-reported cravings for different cues are correlated with each other. We computed Pearson's correlation between each pair of average craving ratings (that is, for food and social cues, measured after fasting, isolation and baseline). These correlations revealed some interesting structures (Supplementary Fig. 5). First, self-reported cravings for food and social interaction were moderately correlated at baseline $(r=0.48)$, but less correlated after either need was deprived (mean $r=0.14, z=1.64, P=0.05$ ). Second, individual differences in cravings for social interaction were somewhat, but not significantly, more stable across the three sessions (mean $r=0.33$ ) than craving for food (mean $r=0.06, z=1.26, P=$ $0.10)$. Finally, the strongest correlation of all was between self-reported craving for food after fasting and for social interaction after isolation $(r=0.64)$. These results suggest that there is a reliable individual difference in craving responses after deprivation, shared across both fasting and isolation, consistent with our observation of a common neural response to both modes of deprivation.

Differences in craving measures.-To explore the differences in the results for the two craving measures (Craving_CIC and Craving_Q ) in more detail, we included the following exploratory analyses: (1) we assessed the correlation between these measures and found that, for both food and social craving, the CIC and $Q$ measures were correlated within session, across individuals (food $r(38)=0.52 ; P<0.001$; social: $r(38)=0.30 ; P<0.032$ ). (2) We tested whether the correlations between craving measures and SN/VTA activity was different for these two measures. We implemented two regression models, one for the fasting session and one for the isolation session. The interaction between SN/VTA activity and craving measure (that is, Craving_CIC and $C_{-}$raving_Q ) and its main effects were entered as predictors. We did not find a significant interaction of craving measure $\times$ SN/VTA activity for social craving ratings $(b=37.65, t=1.03,95 \% \mathrm{CI}=(-35.44,110.74), P=0.31)$ or for food craving ratings $(b=3.16, t=0.11,95 \% \mathrm{CI}=(-58.63,52.31), P=0.91)$. This suggests that for social craving and food craving the correlations between the two ways of measuring craving and SN/VTA activity were not significantly different.

Craving ratings baseline.-Self-reported food craving on fasting days and social contact craving on isolation days were both higher than on the baseline day (Food_Craving_CIC. $t(39)=7.94, P<0.001$, two tailed; Social_Craving_CIC: $t(39)=4.15, P<0.001$, two tailed). Self-reported food craving on the isolation day was lower than on the baseline day $(t(39)=$ $3.59, P=0.001$, two tailed), but self-reported social craving on the fasting day was not lower than on the baseline day $(t(39)=0.64, P=0.526$, two tailed). Supplementary Figure 1 shows the craving ratings for each session including the baseline day. 
Associations between isolation and chronic loneliness.-Participants who reported higher levels of chronic loneliness showed a muted response in the SN/VTA to both social cues $(r(38)=-0.46, P=0.003)$ and food cues $(r(38)=-0.37, P=0.018$, two tailed) during the baseline scan.

Similar to responses after isolation (see "Correlations with chronic loneliness" above), on the baseline day participants higher in chronic loneliness reported less social craving immediately before the scan (Social_Craving_Q, $r(38)=-0.40, P=0.011$, two tailed), although chronic loneliness did not affect craving ratings during the task (Social_Craving_CIC, $r(38)=-0.15, P=0.367$, two tailed).

\section{Discussion}

In humans, acute mandatory social isolation evokes a neural 'craving' response to social cues. Midbrain regions showed selective responses to food cues after fasting and to social cues after isolation. SN/VTA activity was higher in people who self-reported wanting food or social interaction more, after deprivation. The multivariate pattern of SN/VTA response was similar for food and social interaction when craved. People who are forced to be isolated crave social interactions similarly to the way in which a hungry person craves food.

Our findings are consistent with results from the mouse showing that DA neurons in the midbrain represent the neural substrate of social isolation ${ }^{16}$. In mice, DA neurons in the midbrain appear to encode an aversive 'loneliness-like' state that motivates social engagement. Our findings suggest that there is a similar mechanism underlying social craving in humans.

Despite the fact that isolation lasted only $10 \mathrm{~h}$, and the participants knew exactly when it would end, participants reported more loneliness and social craving at the end of the day than they did at the beginning. For people who are highly socially connected, a day of social isolation is a large deviation from typical rates of social interaction. Although when chosen intentionally, solitude can be restful and rejuvenating ${ }^{31,32}$, the externally mandated isolation was subjectively aversive.

Our primary, preregistered hypotheses concerned activity in the SN/VTA. The SN/VTA includes almost exclusively DA neurons, which respond with phasic firing to motivationally relevant cues (see ref. ${ }^{28}$ for review) and is activated by craving for food and drugs of addiction $^{25-27}$. Using a CIC task, we found that the SN/VTA responded more to food cues after fasting and more to social cues after isolation, that is, although participants reported general discomfort and reduced happiness after both fasting and isolation, SN/VTA responses were selective to the deprived cue. The magnitude of the SN/VTA response varied across participants, and was correlated with self-reported craving for the corresponding cue: SN/VTA responses to food cues were correlated with self-reported craving for food (in all sessions), and SN/VTA responses to social cues were correlated with self-reported craving for social contact (also in all sessions). These results fit with the intuitive prediction that the deprivation of a need causes increased craving for the specific need ${ }^{33}$. The specific cravings evoke a generalizable pattern of activity in the SN/VTA: patterns of response to food when 
hungry were more similar to the pattern of response to social cues when isolated than to responses to food when sated. Thus, a common signal at the core of the 'craving circuit' in the SN/VTA responds selectively to motivationally salient deprived cues, independent of their specific content.

By contrast, food and social craving led to dissociable responses almost everywhere else in the brain. Both whole-brain and exploratory ROI analyses revealed that food craving evokes selective responses in the ACC and (less reliably) in the insula and amygdala, but not in the OFC, whereas social craving evokes selective responses in the OFC but not the ACC, insula or amygdala. Even in the striatum, the major target of DA projections from the SN/VTA, craving for food and social contact was spatially dissociated. Fasting enhanced responses to food cues mostly in the NAcc, whereas isolation enhanced response to social cues mostly in the $\mathrm{Ca}$. The $\mathrm{Ca}$ activity during social craving is consistent with prior evidence of caudate activity when people re-live experiences of rejection by a spouse or partner ${ }^{34}$, for meta-analysis. Although social rejection (being deliberately and specifically excluded from social interaction) is conceptually distinct from social isolation (being unable to access social interaction), both rejection and isolation could lead to increased social motivation ${ }^{8}$.

Food craving and social craving thus evoke both shared (SN/VTA) and unshared (striatum, cortical regions) neural responses. One open question concerns how these two kinds of craving might interact, that is, how does inducing food craving affect social motivation, or vice versa?

On the one hand, there is some evidence that deprivation of one need leads to a narrowed focus on the deprived need to the exclusion of other needs. An unexpected finding of our study was that, compared with the baseline (no deprivation) session, neural activity for the nondeprived need was decreased, rather than activity for the deprived need being selectively increased. This pattern of narrowing activity was observed for both food and social cues. For example, the midbrain response to food cues was highest after fasting, slightly but not significantly lower at baseline, and lowest after social isolation and eating to satiety. $\mathrm{Pu}$ and NAcc responses to food cues were the same: not significantly higher after fasting than baseline, but significantly lower after isolation. These results fit with previous fMRI studies that find increased activity in the midbrain and the 'craving circuit' (that is, striatum, OFC, ACC, amygdala and insula) in response to food cues after fasting compared with carefully induced satiety (for example, see refs. ${ }^{25,35,36}$ ), but not reliably when compared with baseline (for example, see refs. ${ }^{37-39}$ ). In the present study, we observed a similar pattern for social craving: midbrain responses to social cues were highest after isolation, slightly but not significantly lower at baseline and lowest after fasting. Thus, at baseline participants' motivation may be spread across multiple sources of reward, and specific acute deprivation may serve to narrow and focus the brain's motivational responses to the deprived target, rather than to enhance it. Depriving one need might thus reduce motivation to pursue other needs. Indeed, there is some evidence that people are less prosocial when hungry ${ }^{40}$, consistent with a reduction in social motivation caused by acute hunger, although see ref. ${ }^{41}$.

On the other hand, there is also some evidence that deprivation of one need leads to increased motivation to pursue other sources of reward. In animal models social isolation 
can cause increased food consumption ${ }^{42}$, increased susceptibility to substance addictions and other general changes in motivational systems ${ }^{8}$, that is, deprivation of social needs can result in generalized reward-seeking behavior, potentially as a form of compensation.

These two possibilities are not mutually exclusive: deprivation could lead to a narrowed focus (reduced pursuit of other needs) or compensation (increased pursuit of other needs), depending on the duration and developmental timing of the deprivation. For example, short-term acute isolation in adults may cause a temporary narrow focus on social connection, whereas long-term or developmental isolation could result in a shut-down of these adaptive efforts, resulting in social withdrawal and other compensatory changes in nonsocial motivation ${ }^{43}$. In our study, people who reported higher levels of pre-existing chronic loneliness showed reduced activity in the SN/VTA in response to social cues, consistent with the idea that chronic isolation can lead to social withdrawal ${ }^{18,44}$ (but see refs. ${ }^{19,20}$ ). However, the causal mechanism underlying the correlation with chronic loneliness remains unclear: it might as well be that individuals with generally reduced sensitivity of motivational brain areas are more prone to becoming lonely. Indeed, people reporting high chronic loneliness in our study also had lower SN/VTA responses to food cues. Chronic loneliness could thus be a consequence, rather than a cause, of general low responsiveness in the SN/VTA.

In addition, the effects of chronic loneliness on social approach motivation might also be mediated via other factors affecting approach behavior more broadly, such as anxiety or depression.

This ambiguity of correlational observations highlights the importance of our design, experimentally inducing acute isolation to disentangle direct effects of isolation as such from individual differences in reward-seeking behavior more generally.

There are two key limitations in the present study that could be addressed in future research. First, we measured craving using passive viewing of craving-related cues and self-report measures of craving, rather than a direct test of motivation, such as participants' willingness to expend effort or money to fulfill a need. Responses in the passive CIC task could also be influenced by low-level processes such as increased visual attention to the deprived category (although the robust prior literature in both humans and animals makes visual attention an unlikely explanation of SN/VTA engagement ${ }^{28}$ ). We used two different measures of self-reported craving and, although these were correlated with each other, different craving measures were significantly correlated with SN/VTA activity for food craving and social craving. Thus, future studies should investigate the changes in subsequent behaviors that are predicted by SN/VTA responses after acute social isolation. Note, however, that, also in domains such as drug addiction and hunger, researchers have struggled to establish a gold-standard behavioral metric of subjective craving with better external validity than self-report ${ }^{45}$.

A second limitation is that we studied a small sample $(n=40)$ of healthy, well-connected young adults, mostly students, in one cultural context. Chronic loneliness disproportionately affects people older (that is, elderly people ${ }^{46}$ ) and younger (that is, adolescents ${ }^{14}$ ) than the 
participants studied here; future studies should test whether the current results generalize to those more vulnerable populations. Yet despite our restricted sample, we observed substantial variability between individuals at baseline, in both self-reported social craving and in SN/VTA response to social cues. We did not measure people's social behaviors at baseline (for example, number and quality of social interactions) or other traits broadly relevant to social interaction (for example, trait extroversion, depression, anxiety). Future studies should investigate how social craving and SN/VTA responses to social cues respond to changes in people's social environment over both short (hours) and medium (weeks or months) time scales, within individuals ${ }^{47}$.

In all, our finding of a more selective SN/VTA response to social cues after isolation, as well as to food cues after fasting, fits the intuitive idea that positive social interactions are a basic human need, and acute loneliness is an aversive state that motivates people to repair what is lacking, similar to hunger. Thus, our research provides empirical support in human participants for the 'social homeostasis' hypothesis developed based on animal models ${ }^{43}$. Despite differences in the duration and setting of social isolation, and in the anatomy of DA midbrain structures, both humans and mice seem to show midbrain craving responses for social interaction, as well as for food. Even this broad similarity of neural responses in mice and humans is encouraging for the translational prospects of mouse models of mental health disorders that affect social motivation—-for example, autism spectrum disorder ${ }^{48}$.

A vital question is how much, and what kinds of, positive social interaction is sufficient to fulfill our social needs and thus eliminate the neural craving response. Technological advances offer incessant opportunities to be virtually connected with others, despite physical separations. Yet, some have argued that using social media only exacerbates subjective feelings of isolation ${ }^{49}$ (but see ref. ${ }^{50}$ ). The potential for virtual interactions to fulfill social needs is particularly relevant when large populations are required to self-isolate, for example during a global pandemic. In early 2020, millions of humans experienced a sudden, externally mandated period of relative or complete physical isolation from others, as public health officials sought to slow the spread of an infectious new coronavirus. This unprecedented upheaval in people's social routines emphasized the need for a better understanding of human social needs and the neural mechanisms underlying social motivation.

\section{Methods}

\section{Participants.}

Participants $(n=40)$ were healthy, right-handed adults, ranging in age from 18 years to 40 years (mean age 26 years; $n=27$ females). An initial power analysis in $\mathrm{G}^{*}$ Power 3.0 (ref. $\left.{ }^{51}\right)$ targeted on the detection of medium effects $(d=0.5, a=0.05$ and $1-\beta=0.80)$ suggested a sample size of $n=34$. The targeted-effect size was chosen based on findings from studies employing CIC paradigms for drug craving ${ }^{52}$, food cravings $55,37,53,54$ and internet gaming craving ${ }^{27}$, which report medium-to-large effect sizes in cue reactivity ${ }^{52}$. We performed power calculations for medium effects because social craving might be less intense or more variable than cue reactivity in drug craving and food craving. We therefore recruited 42 participants to account for potential attrition or exclusion for MRI data quality; 
2 participants were unable to complete all experimental sessions and so were dropped from analysis, leaving 40 complete datasets.

Participants were recruited via email lists and through online advertisements and flyers. Interested individuals filled out a screening questionnaire to assess eligibility for the study (questionnaire data were collected using REDCap Software, v.5.5.17). People were eligible if they reported a healthy BMI (16-30), no current calorie-restricting diet, no permanently implanted metal in their body, no history of brain damage, and no currently diagnosed mental health disorder or substance abuse. As we aimed to study social motivation in a sample of adults who have frequent and regular social interactions, we also excluded people who (1) lived alone, (2) reported current feelings of loneliness on the UCLA loneliness scale ${ }^{21}$ (that is, we excluded people with scores $>50$, which is 1 s.d. above the mean for a student sample ${ }^{21}$ ), or (3) reported smaller social network sizes than typically expected of adults ${ }^{55}$ according to a social network size measure ${ }^{56}$ and the Social Support Questionnaire $^{57}$ (that is, we excluded people with social networks $>2$ s.d. below mean, based on prior measured distributions from Von der Heide et al. ${ }^{58}$ ). All experimental procedures were approved by MIT's institutional review board, COUHES (Committee on the Use of Humans as Experimental Subjects; https://couhes.mit.edu/). Participants signed a consent form describing all experimental procedures before participating in the study. Each participant was compensated with US $\$ 350$ for participating in three fMRI sessions and completing the $10 \mathrm{~h}$ of food fasting and $10 \mathrm{~h}$ of social isolation.

\section{Experimental procedures.}

Each participant was scanned in three fMRI sessions, separated by at least $24 \mathrm{~h}$. Figure 1 shows an overview of the experimental procedures. One session followed $10 \mathrm{~h}$ of food fasting, one session followed $10 \mathrm{~h}$ of social isolation and one session was a baseline without any mandated prior abstinence. All participants underwent all the three experimental conditions (baseline, food fasting and social isolation). Between participants, the order of sessions was counterbalanced; each participant was pseudo-randomly assigned to one of the possible orders of the three different sessions, with the restriction that all six possible sequences were approximately equally likely in the full sample. Data collection was not performed blind to the conditions of the experiments.

Food fasting.-Participants were asked to abstain from consuming any food or drinks/ coffee (except water) for $10 \mathrm{~h}$ before the fMRI session. We scheduled each fMRI session at 19:00; thus, participants were asked to refrain from eating after 9:00 on the day of the fasting session. We followed methods of previous food-craving studies (for a review, see ref. ${ }^{26}$ ) in which participants were instructed to fast at home. Fasting was confirmed through self-report on arrival. We also asked participants to abstain from all forms of exercising on the day of food fasting to avoid exhaustion. Participants filled out an online questionnaire, rating their momentary food craving, hunger, discomfort, happiness and dislike of fasting (on a visual analog scale anchored at 0 (not at all) to 100 (extremely)), every $2 \mathrm{~h}$ during the food-fasting period. Before the scan, participants were asked to select a meal that they would receive after the scan on an online food-ordering platform (Grubhub). Participants selected 
their food while they were still fasted, and we gave no restrictions for what or how much to order.

Social isolation.-Participants were socially isolated for $10 \mathrm{~h}$. On the day of the isolation session, participants arrived at the McGovern Institute for Brain Research, MIT building 46 , at $8.15 \mathrm{am}$. As we aimed to keep all social interactions between the social isolation and the fMRI session to a minimum, participants were given extensive instructions about the paradigm and MRI session, and a mock scanner session, before starting social isolation. Subsequently, participants gave their phones and laptops to the experimenter and were guided to a room containing an armchair, a desk and an office chair, and a fridge with a selection of food, snacks and beverages. Participants remained in that room from 9:00 until 19:00. In advance of the session, participants were invited to send us text documents (without any social content) to read or work on during isolation; approved documents were printed or transferred to the provided laptop. In addition, we provided puzzles, Sudoku, coloring pages, nonsocial games (for example, Tetris, Bubble Shooter) and drawing/writing supplies. Participants were provided with a laptop (with parental controls enabled), allowing them to visit only our Slack channel (that is, an online messenger software allowing communication between a group of people (www.slack.com)) and the webpage containing our online questionnaire. Messaging in Slack was restricted to informing participants about the arrival of food delivery and for emergencies (that is, in case participants ran into problems that required assistance from the research team during isolation). Participants filled out an online questionnaire rating their momentary social craving, loneliness, discomfort, happiness and how much they disliked isolation (visual analog scale anchored at 0 (not at all) to 100 (extremely)) every $2 \mathrm{~h}$ during the social isolation period. The fMRI session was conducted immediately after the social isolation. After the scan, a member of the research team chatted with the participants about their experiences during isolation and made sure participants were not feeling troubled. As living in a shared household was a prerequisite for participating in the experiment, participants were expecting to meet with at least one other person after the experiment.

Baseline.-Participants came into the lab at 19:00 and completed the same fMRI tasks as in the other two conditions (in addition to a functional localizer task, see "Functional localizer task"). Participants were asked not to be hungry at the time of the scan.

\section{Functional MRI.}

Participants were in the scanner for around $1 \mathrm{~h}$ in each session. We started with anatomical scanning. For each participant, structural, whole-head, T2*-weighted structural images were collected in 176 interleaved sagittal slices with 1-mm isotropic voxels (field of view (FOV): $256 \mathrm{~mm}$ ). In addition, whole-head T1-weighted structural images in 176 interleaved sagittal slices with 1-mm isotropic voxels (FOV: $256 \mathrm{~mm}$ ) were collected. The T2*-weighted anatomical scan was collected for anatomical identification of midbrain nuclei (that is, the high content of iron in SN/VTA and red nucleus makes the T2* shorter and darker in these areas ${ }^{28,59}$ ). We confirmed the identification of midbrain structures by registering with the newly available atlas of subcortical nuclei from Pauli et al. ${ }^{60}$ and defined separate ROIs in the dorsal and ventral striatum. We also collected a field map (phase-difference B0 
estimation; echo time $1(\mathrm{TE} 1)=3.47 \mathrm{~ms}$, echo time $2(\mathrm{TE} 2)=5.93 \mathrm{~ms})$ to control for spatial distortions, which are particularly problematic in midbrain fMRI ${ }^{28,59}$. During acquisition of the anatomical images and the field map ( $15 \mathrm{~min}$ in total) participants lay quietly in the dark.

Subsequently, we collected functional data during six runs of a CIC task (see "CIC task" for details). Each functional run consisted of 147 volumes with $58 \mathrm{~T} 2 *$-weighted echo planar slices $(\mathrm{TR}=2,000 \mathrm{~ms}, \mathrm{TE}=30 \mathrm{~ms}, \mathrm{FOV}=210 \mathrm{~mm}, 70 \times 70$ matrix, yielding a voxel size of $3 \times 3 \times 3 \mathrm{~mm}^{3}$ ) acquired as a partial-head volume in an anteroposterior phase-encoding direction using interleaved slices. The scanning parameters were extensively piloted ( $n=$ 11) using the functional localizer task (see "Functional localizer task") and the parameters showing the best signal-to-noise ratio were selected for the study. Despite the small structure of the SN/VTA, we thus chose 3-mm isotropic voxels because of their higher signal-to-noise ratio compared with smaller voxels ${ }^{61}$. The angle of the slices was approximately $20^{\circ}$ away from the plane of the standard anterior commissure-posterior commissure to avoid placing the SN/VTA and the sphenoid sinus in the same slice plane. This reduced geometric distortion to the point that standard distortion correction methods could be applied ${ }^{28}$. The CIC paradigm took approximately $26 \mathrm{~min}$ in total.

CIC task.-We designed a new CIC task to simultaneously measure craving for both food and social interaction, relative to a control. Participants viewed colored images depicting: (1) groups of individuals as they meet, talk, laugh, smile, etc.; (2) different kinds of highly palatable foods such as cake, pizza, chocolate, etc.; and (3) attractive flowers as the control condition.

On each trial, participants saw a single photograph and a three- to five-word verbal description, for $5 \mathrm{~s}$. The combination of visual and verbal cues was intended to maximize deep semantic processing of the relevant attributes. Each trial was followed by a 1-s rest period (during which a fixation cross was displayed). Three consecutive trials were presented in a block of the same condition (food, social, control). Each block was followed by a jittered 2- to 6-s rest period. Subsequently, participants self-reported how much they were currently craving food (on food blocks) or social interaction (on social blocks). After control blocks, participants rated how much they liked the flower image, to match the demand for response preparation. A second jittered 2- to 6-s rest period preceded the onset of the next block. In total, participants saw 18 blocks (54 trials) per condition, per scan session. The trials on each day were unique, so in total participants saw 36 blocks (108 unique images with descriptions) per condition. The duration of the task was approximately $30 \mathrm{~min} —$ divided into six runs, with each run having a duration of approximately $5 \mathrm{~min}$.

The stimuli for the CIC task were tailored to each individual's preferred foods and modes of social interaction. During the initial screening, participants were asked to list their top ten favorite foods and social activities. Stock photographs illustrating these specific foods and activities were selected from a large public database (https://www.pexels.com/), and then verbal labels were added using the participant's own descriptions. Food descriptions included 'fluffy syrup-drenched pancakes', 'creamy cheesy macaroni', 'refreshing mixed fruit salad' and 'yummy vanilla cake with sprinkles'. Social descriptions included 'chatting 
and laughing together', 'joking around with friends', 'supporting each other through workouts' and 'enjoying a conversation together'. Social pictures were all matched for gender of participants (that is, for a male participant, all social photographs included at least one man). The stimuli were images of strangers, rather than images of the participant's own friends and family, to (1) match the food and control images for novelty, because SN/VTA activity is sensitive to novelty ${ }^{62}$, (2) match image quality across conditions and participants, and (3) avoid unmeasured variability in the quality or current status of participants' relationships with specific individuals. Control trials presented attractive photographs of flowers accompanied by positive valence verbal descriptions (Fig. 1). For group-level results in response to cues (across sessions), see Supplementary Table 23 and Supplementary Fig. 6.

Functional localizer task.-During the baseline session, each participant completed a functional localizer at the end of the scan. We anticipated that anatomical localization of SN/VTA might be difficult, given the strong susceptibility to magnetic distortions in the midbrain ${ }^{28,59}$. We therefore designed a task to functionally identify relevant midbrain regions in each participant individually. The task was an adapted version of Krebs et al. ${ }^{62}$. As midbrain DA neurons in the SN/VTA respond to both novelty and reward ${ }^{62-64}$, we contrasted new and rewarding stimuli against familiar and nonrewarding stimuli. However, our preregistered hypotheses focus on the anatomical localization strategy, so we treat analyses of the functionally localized regions as exploratory.

A potential limitation of this approach is that it was targeting voxels responding to secondary reinforcers (that is, money) rather than primary reinforcers (such as food and social contact). In animal models, dopamine neurons in the midbrain exhibit burst firing in response to both primary reinforcers and conditioned stimuli once the conditioning has been established (for example, see refs. ${ }^{65,66}$ ). Conditioned stimuli are somewhat less effective than primary rewards in terms of response magnitude and fractions of neurons activated ${ }^{67}$. Thus, by localizing the SN/VTA based on activity in response to anticipated financial rewards, we may have identified only a subset of voxels that respond to primary rewards. Importantly, both food and social cues would be similarly affected, so our key claim of similarity between these responses would not be undermined. In addition, our functional localization method was complementary to an anatomical ROI, which would not be affected by this issue.

Before beginning the localizer task, participants memorized a set of five images depicting abstract art (all images taken from the free stock pictures site (https://www.pexels.com)). During the task, the abstract art images served as cues to the condition of the current trial. The task had two conditions: a reward/loss condition (reward) in which participants could earn or lose money depending on whether their responses were correct and fast enough, and a nonreward condition (nonreward) in which participants always received US $\$ 0$ regardless of their response. Each trial started with an abstract art image. The previously memorized (familiar) images indicated a nonreward trial. Abstract art images that were not previously observed (new) indicated a reward trial. After the cue, participants saw a number between 1 and 9 (excluding 5) for $100 \mathrm{~ms}$ on the screen. Their task was to press an assigned button indicating whether the number is $<5$ or $>5$ as fast as possible. Initially, correct responses were required in <500 ms; after 10 consecutive correct answers, this window was reduced 
to $400 \mathrm{~ms}$. After they pressed the button, participants saw the outcome indicating whether they won US $\$ 1$ (reward trial, correct response, fast enough), lost US $\$ 0.20$ (reward trial, wrong response or too slow) or received US $\$ 0$ (nonreward trial). In total, participants played 80 trials (40 trials per condition) and the duration of the task was approximately $10 \mathrm{~min}$. Participants responded correctly and within the time limit on $87 \%$ of reward trials and $69 \%$ of nonreward trials. The earnings from this task were added to participants' compensation after the baseline session. This design allowed us to compare responses to new stimuli predicting reward versus familiar stimuli predicting no possibility of reward. For group-level results within the midbrain in response to reward > nonreward, see Supplementary Fig. 7.

\section{Behavioral data analysis.}

Questionnaire data.-For each participant we collected two measures of social network size (that is, number of monthly interactions and number of close relationships). These scores indicate the size of participant's social network on different hierarchical levels ${ }^{55}$. However, because the measures were highly correlated $(r(39)=0.58, P<0.001)$, we $z$-transformed and averaged the two measures for each participant. This gave us an indicator of participant's social network size relative to the sample's average social network size. In addition, we calculated a loneliness score for each participant using the UCLA loneliness scale $^{21}$. We tested how self-reported ratings of hunger, food craving, discomfort, happiness and dislike of fasting provided during food fasting changed over the course of $10 \mathrm{~h}$ using paired Student's $t$-tests comparing the first rating (collected at the start of fasting) and last rating (collected after $10 \mathrm{~h}$ of fasting). We used the same analysis for the ratings provided during social isolation: loneliness, social craving, discomfort, happiness and dislike of isolation. Data distribution was assumed to be normal but this was not formally tested. Three participants missed filling out the first or last round of the online questionnaire during fasting, so statistical analyses involving this questionnaire were conducted with a sample size of $n=37$.

\section{Data analysis of fMRI.}

Preprocessing.-We used open source preprocessing pipelines for fMRI data, developed through the nipy and nipype ${ }^{68}$ initiatives. We used the heudiconv python application which uses dcm2niix to convert raw scanner data into the NIFTI image file format, then organizes these data into a BIDS-formatted directory structure. The FMRIPrep application ${ }^{69}$ was used to minimally preprocess the anatomical and functional data (using default settings but including susceptibility distortion correction using fieldmaps). Using FMRIPrep, we skull-stripped anatomical images first roughly using the atlas-based ANTS program ${ }^{70}$, and then refined it using information from FreeSurfer surfaces after reconstruction was completed $^{71}$. Brain tissue segmentation was performed with the FMRIB Software Library (FSL) FAST program ${ }^{72}$. Images were spatially normalized to 2-mm isotropic MNI-space using the multiscale, mutual-information-based, nonlinear registration scheme implemented in ANTS. We visually inspected brain masks, tissue segmentation and FreeSurfer surfaces. Susceptibility distortion correction was performed using phase-difference B0 estimation ${ }^{73}$.

A reference image for each run was generated from the input BOLD timeseries. A functional brain mask was created using a combination of FSL, ANTS, AFNI and nilearn tools ${ }^{74}$. 
Using FSL's MCFLIRT program ${ }^{75}$, we estimated and corrected for head motion, resulting in a coregistered BOLD series as well as motion-based confound regressors. Any run containing a framewise displacement $>0.4 \mathrm{~mm}$ on $>25 \%$ of the total frames was excluded from additional analyses. Additional confound regressors were generated, including other measures of motion (framewise displacement and DVARS and anatomical CompCor ${ }^{76}$ timeseries derived from cerebrospinal fluid and white matter tissue segments). The reference image of each run was aligned with the anatomical image using FreeSurfer's program 'bbregister' 77 . The timepoint-to-functional reference transformation, the functional reference to anatomical transformation and the anatomical-to-MNI transformation were concatenated into a single transformation, and used to transform each functional timeseries into MNI template space. Spatial smoothing was performed on the FMRIPrep outputs with a 6-mm smoothing kernel using FSL's SUSAN tool ${ }^{78}$, which uses segmentation boundaries to avoid smoothing across tissue types. MRIQC, an open source quality assurance software tool ${ }^{79}$, was used to generate additional reports that display image quality metrics.

Modeling.-Analyses were conducted using the nipype framework ${ }^{68}$. For run-level analyses, the preprocessed timeseries was assessed with algorithms from the Artifact Removal Toolbox (ART) ${ }^{80}$ to identify frames within the run that have an abnormal amount of motion $(0.4 \mathrm{~mm}$ of total displacement, or an intensity spike $>3$ s.d. from the mean). The design matrix included boxcars for the experimental conditions convolved with a double-gamma hemodynamic response function (HRF), and nuisance regressors representing framewise motion, the anatomical CompCor regressors derived from white matter and cerebrospinal fluid, as well as impulse regressors for volumes identified by ART. A high-pass filter $(120 \mathrm{~Hz})$ was applied to the design matrix and the smoothed data. The model was evaluated using FSL's FILM program ${ }^{81}$. Subject-level contrast maps were generated using FSL's FLAME ${ }^{81}$ in mixed-effects mode.

Data exclusion.-Exclusion criteria were pre-established in our preregistration (https:// osf.io/cwg9e). We excluded three runs of fMRI data from the overall sample (from two participants from the CIC task) based on the following criterion: any run containing a framewise displacement $>0.4 \mathrm{~mm}$ on $>25 \%$ of the total frames will be excluded from additional analyses.

ROI definition.-We included functional voxels that overlapped at least $75 \%$ with the SN and the VTA region (no. of voxels = 161) from the probabilistic atlas of human subcortical nuclei ${ }^{60}$. As the striatum is a major target of projections from midbrain neurons, and their firing results in increased DA transmission in the striatum ${ }^{30,82,83}$, we expected to see the same pattern of results in the striatum, that is, increased activation to food cues after food deprivation and to social cues after social isolation, and a positive correlation between activity in striatum and self-reported craving (for both food and social craving). Thus, we also included three additional ROIs in our analysis: $\mathrm{Pu}$ (no. of voxels $=2,530$ ), $\mathrm{Ca}$ (no. of voxels $=2,523$ ) and NAcc (no. of voxels $=300$ ) also using the probabilistic subcortical atlas ${ }^{60}$. 
Functional ROI definition.-To define subject-specific ROIs, we used individual activations of each participant in the localizer task. The fMRI timeseries was analyzed using an event-related design approach implemented in the context of the GLM. The model contained two regressors separately modeling the presentation of new/reward cues, and familiar/nonreward cues (that is, when the abstract art images were presented, $2 \mathrm{~s}$ ). We also included one regressor for the time period of button press and outcome (1.1 s). As we did not add any jitter between button press and presentation of outcome (because this was not the contrast of interest), we modeled the whole segment as one block. For each participant, we calculated the target contrast new reward > familiar nonreward. We then used a mask encompassing the whole midbrain as the search space for the selection of individual voxels. In each participant we selected the top 100 active voxels within the search space in response to the target contrast.

\section{Spatial and functional differences in anatomical and functional ROIs.-We} explored the overlap in voxels between functional and anatomical SN/VTA ROIs in each participant and found that it was variable across participants, with the maximum overlap being approximately one-third of the voxels. Supplementary Figure 8 shows a histogram of the overlap between the two masks across subjects. On the other hand, these two methods are probably measuring a similar underlying function because the mean activity in response to social cues after isolation was highly correlated across subjects $(r(38)=0.81 ; P<0.001)$, as shown in the scatterplot in Supplementary Fig. 9. Thus, we interpret the difference in results between functional and anatomical masks as reflecting the uncertainty of the measurement.

Definition of exploratory ROls.-We selected ROIs based on converging results from five meta-analyses of craving across different modalities ${ }^{26,84-86}$ identifying signals of craving in the OFC, amygdala, ACC and insula. As the meta-analyses report varying coordinates for the foci of activation, we chose to create ROIs based on an anatomical atlas instead (that is, Harvard-Oxford cortical and subcortical probabilistic anatomical atlases; included in the FSL). We selected all voxels that showed a minimum probability of 50\% of being in the respective area, and extracted the mean activity from those voxels in response to food, social and control cues for each session (fasting, baseline and isolation). We then calculated a mixed-model regression for each region within this 'craving circuit' (and reported results as significant at $P<0.0125(0.05 / 4)$ ). In addition, due to previous findings showing that the ventral striatum and NAcc are activated during craving for food and drugs ${ }^{85}$ and associated with altered responses in chronic loneliness ${ }^{18,20}$, we explored this region further at the suggestion of a reviewer by selecting the top 100 voxels within the NAcc that were active in response to the midbrain localizer task (that is, active in response to reward anticipation) and assessed whether these voxels code for food and social craving (see Supplementary Materials for results).

Univariate analyses.-For our planned analyses, we used mixed-effects regressions (using Matlab 2019b's fitlme function) to estimate the fixed effects of cue, deprivation session, and the critical interaction of cue and deprivation session, on response magnitude in the ROIs, controlling for each session's average framewise displacement (that is, head 
motion), with the subject included as a random effect. Following the recommendation of Barr et al. ${ }^{87}$, we used maximal models, including participants as a random effect with both random intercepts and random slopes. Data distribution was assumed to be normal but this was not formally tested. We modeled these effects in the anatomically defined SN/VTA (preregistered analysis) and in the functionally defined ROI of voxels maximally sensitive to reward and novelty (exploratory analysis). Thus, for each ROI, we tested two mixed-effects models:

Target comparison: fasting versus isolation.: First, we tested our key hypothesis that responses to food cues (relative to flowers (control)) would be higher after fasting, and responses to social cues (relative to control) would be higher after isolation. In this model, the fixed effects were: session (fasting versus isolation; contrast coded), cue (control, + food, +social; indicator coded), the interactions of cue and session, and mean framewise displacement in that session; we also included random intercepts and random slopes for each participant.

Exploratory analyses: baseline session.: To test whether responses were different on the deprivation days, compared with the baseline day, we modeled responses on all three sessions by including fixed effects of session (baseline, +fasting, +isolation; indicator coded), cue (control, +food, +social; indicator coded), the interactions of cue and session, and mean framewise displacement in that session; we also included random intercepts and random slopes for each participant.

The command for both models was:

$$
\text { fitlme(Data, 'Response }(\operatorname{session} * \text { cue })+(\text { MeanFD })+(\text { session*cue } \mid \text { subjectID }) \text { '). }
$$

To test whether these responses were correlated with individual differences in self-reported craving, we calculated the average contrast value (food > flowers and social > flowers) in the anatomically defined SN/VTA for each participant. We used two different approaches to measure participants' self-reported craving. First, we calculated the mean craving rating participants reported on each trial during the CIC task in the scanner (Craving_CIC). This measure was exactly comparable across sessions and simultaneous with the fMRI data acquisition. As the data for food craving were truncated at the upper limit of 10 (Fig. 3c), we also calculated a truncated regression model (using the truncreg package in $\mathrm{R}$ ) using the truncated variable as the explanatory variable in the regression model ${ }^{88,89}$ in addition to the standard correlation. Second, we took the craving reported by participants on the final online questionnaire, completed after $10 \mathrm{~h}$ of fasting or isolation (Craving_Q). On this measure, participants reported craving only for the deprived need (food when fasting, social contact when isolated), but this measure provides the most direct measure of the effect of deprivation because it can be compared with the self-report at the start of each session. In addition, the Craving_ $Q$ ratings were completed on a finer scale (0-100 rather than $0-10)$ and participants had no time restriction when filling out the questionnaires (although, during the task, participants had $5 \mathrm{~s}$ to complete the scales). For these reasons, we included both types of craving ratings and report results as significant at $a<0.025$. We measured 
correlations between self-reported craving and neural responses for each deprivation session. As we specifically predicted a positive correlation between craving for the deprived target and response magnitude in the SN/VTA, these correlations were tested one tailed. Data for the analyses were extracted using FSL's 'fslmeants' utility and subsequent univariate and correlation analyses were conducted in Matlab 2019b, RStudio (1.1.423) and SPSS 26.

Multivariate analyses.-We next used multivoxel pattern analysis to determine whether the multivariate spatial pattern of activity in SN/VTA is shared for food and social craving. From the generalized linear model, we extracted the $\beta$ values (that is, amplitude of the fit HRF) of the response to each condition (food cues, social cues, flower cues) for each block in each run ( 3 blocks per condition per run $\times 6$ runs) in each session (baseline, fasting, isolation) resulting in $162 \beta$ values for each voxel. Responses were extracted from all voxels in the anatomically identified SN/VTA in each participant (that is, no additional feature selection was applied). All multivariate analyses were conducted with the PyMVPA ${ }^{90}$ toolbox in Python (http://www.pymvpa.org) and Matlab 2019b. Although smoothing has been shown not to substantially affect information in multivariate data ${ }^{91-93}$, because of the small size of the SN/VTA, we also ran the classification analyses on unsmoothed data and found that we were not able to decode stimulus or motivational state across sessions in the unsmoothed data (see "Additional analyses").

First, as a proof of concept, we tested whether we could classify food cues from control cues from the SN/VTA within the food-fasting session (see "Additional analyses"). Then, we trained a linear support vector machine classifier, training using all $36 \beta$ values ( 2 cues $\times 3$ blocks $\times 6$ runs) of the food-fasting session to discriminate patterns of responses to food versus flower cues. We tested the generalization of the classifier to responses to social versus flower cues in the isolation session (that is, different category of cue but similar motivational state), producing an accuracy score (correct classification for $36 \beta$ values). If social craving and food craving share a neural basis, we predicted that a classifier trained on food_craved versus control cues would successfully (above chance) classify social_craved versus control cues. We also tested the generalization of this classifier to food versus flower cues, in the isolation and baseline sessions (that is, the same categories of cues as the training, but different motivational state), and to social versus flower cues at baseline (that is, different category and different motivational state, as a control analysis). To obtain confidence intervals of the mean in the data samples, we used bootstrapping. We generated 1,000 datasets randomly by sampling with replacement from the classification accuracies across participants using Matlab's bootci function.

For hypothesis testing on the group level, we used a permutation analysis following the methods in Stelzer et al. ${ }^{94}$. This nonparametric approach does not depend on assumptions about the distribution of classification accuracies ${ }^{94,95}$. To generate a null distribution from the data, we followed the steps described in Stelzer et al., which we summarize below and visualize in Fig. 4. We shuffled the condition labels randomly during training within each run, and then tested the prediction accuracies for each cross-classification on the test data. We performed this permutation analysis 100 times per participant (thus creating 100 random permutations), resulting in 100 accuracy values per participant, for each testing dataset. To create a null distribution on the group level, we then randomly drew one of the 100 accuracy 
values for each participant, and calculated a mean across participants. This procedure was repeated $10^{5}$ times for each testing dataset, creating the null distributions for each dataset (see Fig. 4 for the histograms showing the null distributions). We calculated the probability $P$ of obtaining a mean accuracy value in the null distributions that is equal to or higher than the true mean from the analyses. Following Stelzer et al., we rejected the null hypothesis of no group-level decoding if $P<0.001$, which corresponds to a low probability if it were retrieved by chance.

Finally, we used representational similarity analysis to test which pattern of activity is more similar to 'food_craved': social_craved or food_noncraved. We predicted that the presence of a craved object should be more important for SN/VTA activity than the cue category, so we predicted that the pattern of food_craved responses will be more similar to the pattern of response to social_craved cues, compared with when these cues are presented in different motivational states (social_craved $\times$ food_noncraved) or even the same cue in different motivational states (food_craved $\times$ food_noncraved). We averaged responses to each cue across all six runs per session to obtain one mean $\beta$ value for each voxel. Then we calculated the dissimilarity ( 1 - Pearson's correlation, Fisher transformed) between each average response pattern. Then we compared the dissimilarity between food_craved $\times$ social_craved (that is, food fasted and social isolated; different stimuli but similar motivational state) to the dissimilarity between food_noncraved $\times$ social_craved (that is, food baseline and social isolate; maximally distant (different stimuli, different motivational state and different session — the noise ceiling)) and to the dissimilarity between food_noncraved $\times$ food_craved (that is, food isolation and food fasted; same stimuli but different motivational state; we used isolation instead of baseline as it represents the satiated control condition to fasting); both comparisons used pair-wise Student's $t$-tests. Data distribution was assumed to be normal but this was not formally tested.

Correlations univariate fMRI measures and behavioral data.-In follow-up analyses, we tested whether individual differences in social network size predict the magnitude of self-reported and neural measures of social craving. We used the UCLA loneliness score obtained from each participant as an indicator of their chronic loneliness. We correlated these two measures with mean values extracted from the midbrain and striatum ROIs from the contrast social_craved >control.

\section{Additional analyses.}

SN/VTA: multivariate decoding of food cues.-As a proof of concept, we tested whether we could discriminate fMRI patterns between food and control cues in the fasting session in the SN/VTA. For each participant, we partitioned the data into six independent folds (six runs), and iteratively trained a linear support vector machine classifier on five runs (that is, fifteen $\beta$ estimates per condition) and tested on the left-out run (three $\beta$ estimates per condition). We then averaged the classification accuracy across runs to yield a single estimate for each participant. This within-session classification tested whether we would be able to decode the cue type (food versus control) from multivariate patterns within the SN/ VTA. We tested whether the accuracy was significantly above chance using bootstrapping. We first generated a null distribution of the data by shifting the mean to be 0.5 (by 
calculating dataset - mean(dataset) +0.5 ), and then generated 1,000 datasets randomly by sampling with replacement from the classification accuracies across subjects using Matlab's bootstrap function. We then calculated a mean for each of the 1,000 simulated datasets from the null distribution and compared it with our observed data average. We calculated the probability $P$ of obtaining a mean value across the 1,000 datasets that is equal or higher than the actual mean from the original dataset, and rejected the null hypothesis of no group-level decoding if $P<0.05$. For obtaining confidence intervals of the mean in the data samples, we generated 1,000 datasets randomly by sampling with replacement from the classification accuracies across subjects using Matlab's bootci function. We found above-chance (50\%) decoding of food cues from flower images after fasting (mean accuracy $=0.5556 ; P<0.001$, $95 \% \mathrm{CI}=0.527-0.585)$.

SN/VTA: cross-classification of food and social cues-unsmoothed data.-We ran the same analysis as reported in the section "Multivoxel pattern analysis" on data for which we did not perform any smoothing during preprocessing to test whether smoothing has substantial effects on our results. First, we tested how smoothing affected the accuracy to discriminate fMRI patterns between food and control cues in the fasting session in the SN/ VTA, using the same method as described in SN/VTA: multivariate decoding of food cues on the unsmoothed data. In the present study, we found that omission of smoothing showed slightly lower mean accuracy across subjects (mean accuracy $=0.542$ ), but still resulted in successful above chance $(50 \%)$ decoding of food cues from flower images after fasting $(P$ $=0.002,95 \% \mathrm{CI}=0.513-0.570)$. Then, as in the original analysis, we trained a classifier on the pattern of activity in the SN/VTA after fasting to food cues and flower images, and tested whether it would successfully decode food and social cues from control cues in the other two sessions (baseline and isolation). Again, we generated null distributions using the permutation analysis described in the main text (following Stelzer et al. ${ }^{94}$ ). In the unsmoothed data, we found no above-chance decoding ( $a=0.001)$ of social cues from flowers after isolation (mean accuracy $=0.521$, bootstrapped $\mathrm{CI}=(0.503,0.542), P=0.041)$ or after baseline (mean accuracy $=0.526$, bootstrapped $\mathrm{CI}=(0.501,0.546), P=0.021)$. In addition, in unsmoothed data, the classifier was also unable to decode food cues from flowers after isolation and baseline (isolation: mean accuracy $=0.522$, bootstrapped $\mathrm{CI}=$ $(0.500,0.544), P=0.021$; baseline: mean accuracy $=0.525$, bootstrapped $\mathrm{CI}=(0.501,0.557)$, $P=0.022)$.

Exploratory whole-brain group analyses.-As a complementary analysis, we assessed the main effects and interactions of cue and session on whole-brain activity. We set up a flexible factorial model with the factors subject, session (baseline, fasting, isolation) and cue (food, social). We entered the first-level contrasts food > control and social > control for each session into the group-level analysis. Analyses were implemented in SPM12b.

Main effects: cue.: We first assessed whole mean brain activity for the contrasts food > control and social > control across all experimental sessions (baseline, fasting, isolation) as a manipulation check to assess which regions were activated in response to food (versus control) and social (versus control) cues. We corrected for multiple comparisons using 
whole-brain family-wise error correction. Supplementary Table 21 and Supplementary Fig. 7 show the results from this analysis.

Interaction effects: cue $\times$ deprivation.: We then the assessed the effects of isolation on brain activity in response to social cues and the effects of fasting on brain activity in response to food cues in the whole brain. To do this, we calculated two contrasts: (1) food: fasting > isolation and (2) social: isolation > fasting. These analyses were exploratory and statistical inference was performed using a threshold of $P<0.05$ corrected for multiple comparisons over the whole brain, using the Gaussian random fields approach at cluster level with a voxel-level intensity threshold of $P<0.001$ (ref. ${ }^{96}$ ). We note that this method of correction for multiple comparisons is probably biased toward false positives ${ }^{97,98}$. We decided to share these lenient analyses (Supplementary Tables 22 and 23 and Supplementary Fig. 8) to be more complete and for potential future meta-analyses.

Midbrain localizer: group analysis._As described in the section "Functional ROI definition" above, we used a functional localizer to select the most active voxels for reward anticipation and novelty within the midbrain to functionally localize the SN/VTA in each participant individually. To explore the localization of midbrain responses to the localizer task in more detail, we implemented a group random effects analysis, entering the first-level contrast reward > nonreward from each participant (Supplementary Fig. 9)

Data analysis was not performed blind to the experimental conditions.

\section{Supplementary Material}

Refer to Web version on PubMed Central for supplementary material.

\section{Acknowledgements}

This research was carried out at the Athinoula A. Martinos Imaging Center at the McGovern Institute for Brain Research at MIT. We thank K. Sottilare, M. Humphreys, J. Huettig, R. Ezzo, J. Weddington, J. Kennedy, M. Hung and I. Nichoson for help with data collection and thank D. Tamir, J. Mildner, H. Richardson, S. Liu, E. Duzel, N. Kanwisher and D. Nettle for advice and discussion. We also thank A. Gupta for his help making the fMRI dataset publicly available. Support for this project came from an SFARI Explorer Grant from the Simons Foundation (grant no. 597310 to R.S.), a MINT grant from the McGovern Institute (grant no. 1496911 to R.S.), an NIH Pioneer Award (no. DP1-AT009925 to K.M.T.), a Max Kade Foundation fellowship (to L.T.), an Erwin Schroedinger Fellowship by the Austrian Science Fund (no. J4326 to L.T.) and an NIH shared instrumentation grant (no. 1S10OD021569-01). R.S. participated in the Center for Brains, Minds and Machines, funded by an NSF STC award (CCF-1231216).

\section{References}

1. Hawkley LC \& Cacioppo JT Loneliness and pathways to disease. Brain Behav. Immun 17, S98S105 (2003). [PubMed: 12615193]

2. Leigh-Hunt $\mathrm{N}$ et al. An overview of systematic reviews on the public health consequences of social isolation and loneliness. Public Health 152, 157-171 (2017). [PubMed: 28915435]

3. Cacioppo JT, Hawkley LC \& Thisted RA Perceived social isolation makes me sad: 5-year crosslagged analyses of loneliness and depressive symptomatology in the Chicago Health, Aging, and Social Relations Study. Psychol. Aging 25, 453-463 (2010). [PubMed: 20545429]

4. Wang J et al. Social isolation in mental health. Soc. Psychiatry Psychiatr. Epidemiol 52, 1451-1461 (2017). [PubMed: 29080941] 
5. Cacioppo JT, Cacioppo S \& Boomsma DI Evolutionary mechanisms for loneliness. Cogn. Emot 28, 3-21 (2014). [PubMed: 24067110]

6. Baumeister RF \& Leary MR The need to belong: desire for interpersonal attachments as a fundamental human motivation. Psychol. Bull 117, 497-529 (1995). [PubMed: 7777651]

7. Bhanji JP \& Delgado MR The social brain and reward: social information processing in the human striatum. Wiley Interdiscip. Rev. Cogn. Sci 5, 61-73 (2014). [PubMed: 24436728]

8. Tomova L, Tye K \& Saxe R The neuroscience of unmet social needs. Soc. Neurosci 10.1080/17470919.2019.1694580 (2019).

9. Evans MJ et al. Social reinforcement of operant behavior in rats: a methodological note. J. Exp. Anal. Behav 62, 149-156 (1994). [PubMed: 8064210]

10. Hiura LC, Tan L \& Hackenberg TD To free, or not to free: social reinforcement effects in the social release paradigm with rats. Behav. Process 152, 37-46 (2018).

11. Angermeir WR Some basic aspects of social reinforcement in albino rats. J. Comp. Physiol. Psychol 53, 364-367 (1960).

12. Novick AM et al. The effects of early life stress on reward processing. J. Psychiatr. Res 101, 80-103 (2018). [PubMed: 29567510]

13. Hall FS Social deprivation of neonatal, adolescent, and adult rats has distinct neurochemical and behavioral consequences. Crit. Rev. Neurobiol 12, 129-162 (1998). [PubMed: 9444483]

14. Orben A, Tomova L \& Blakemore S-J The effects of social deprivation on adolescent development and mental health. Lancet Child Adolesc. Health 10.1016/S2352-4642(20)30186-3 (2020).

15. Panksepp J \& Beatty WW Social deprivation and play in rats. Behav. Neural Biol 30, 197-206 (1980). [PubMed: 7447871]

16. Matthews GA et al. Dorsal raphe dopamine neurons represent the experience of social isolation. Cell 164, 617-631 (2016). [PubMed: 26871628]

17. Volkow NoraD. \& Morales M The brain on drugs: from reward to addiction. Cell 162, 712-725 (2015). [PubMed: 26276628]

18. Cacioppo JT, Norris CJ, Decety J, Monteleone G \& Nusbaum H In the eye of the beholder: individual differences in perceived social isolation predict regional brain activation to social stimuli. J. Cogn. Neurosci 21, 83-92 (2009). [PubMed: 18476760]

19. D'Agostino AE, Kattan D \& Canli T An fMRI study of loneliness in younger and older adults. Soc. Neurosci 14, 136-148 (2019). [PubMed: 29471719]

20. Inagaki TK et al. Yearning for connection? Loneliness is associated with increased ventral striatum activity to close others. Soc. Cogn. Affect. Neurosci 11, 1096-1101 (2016). [PubMed: 26084531]

21. Russell DW UCLA Loneliness Scale (Version 3): reliability, validity, and factor structure. J. Personal. Assess 66, 20-40 (1996).

22. Cacioppo JT et al. Lonely traits and concomitant physiological processes: the MacArthur social neuroscience studies. Int. J. Psychophysiol 35, 143-154 (2000). [PubMed: 10677643]

23. Dickinson A \& Dearing MF Appetitive-aversive interactions and inhibitory processes. In Mechanisms of Learning and Motivation: A memorial volume to Jerzy Konorski (eds Dickinson A \& Boakes RA) 203-231 (Erlbaum, 1979).

24. Matsumoto M \& Hikosaka O Two types of dopamine neuron distinctly convey positive and negative motivational signals. Nature 459, 837-841 (2009). [PubMed: 19448610]

25. Malik S, McGlone F, Bedrossian D \& Dagher A Ghrelin modulates brain activity in areas that control appetitive behavior. Cell Metab. 7, 400-409 (2008). [PubMed: 18460331]

26. Pursey KM et al. Neural responses to visual food cues according to weight status: a systematic review of functional magnetic resonance imaging studies. Front. Nutr 1, 7 (2014). [PubMed: 25988110]

27. Zhang JT et al. Effects of craving behavioral intervention on neural substrates of cue-induced craving in Internet gaming disorder. Neuroimage Clin. 12, 591-599 (2016). [PubMed: 27699148]

28. Duzel E et al. Midbrain fMRI: applications, limitations and challenges. in fMRI: From Nuclear Spins to Brain Functions (eds Uludağ K, Uğurbil K \& Berliner L) 581-609 (Springer, 2015).

29. Berridge KC \& Robinson TE Parsing reward. Trends Neurosci. 26, 507-513 (2003). [PubMed: 12948663] 
30. Schott BH et al. Mesolimbic functional magnetic resonance imaging activations during reward anticipation correlate with reward-related ventral striatal dopamine release. J. Neurosci 28, 1431114319 (2008). [PubMed: 19109512]

31. Nguyen T.-vT., Ryan RM \& Deci EL Solitude as an approach to affective self-regulation. Personal. Soc. Psychol. Bull 44, 92-106 (2018).

32. Thuy-vy TN, Werner KM \& Soenens B Embracing me-time: motivation for solitude during transition to college. Motiv. Emot 43, 571-591 (2019).

33. Hull CL Principles of Behavior: An Introduction to Behavior Theory (Appleton-Century, 1943).

34. Cacioppo $\mathrm{S}$ et al. A quantitative meta-analysis of functional imaging studies of social rejection. Sci. Rep 3, 2027 (2013). [PubMed: 24002359]

35. Haase L, Cerf-Ducastel B \& Murphy C Cortical activation in response to pure taste stimuli during the physiological states of hunger and satiety. Neuroimage 44, 1008-1021 (2009). [PubMed: 19007893]

36. Mohanty A, Gitelman DR, Small DM \& Mesulam MM The spatial attention network interacts with limbic and monoaminergic systems to modulate motivation-induced attention shifts. Cereb. Cortex 18, 2604-2613 (2008). [PubMed: 18308706]

37. Goldstone AP et al. Fasting biases brain reward systems towards high-calorie foods. Eur. J. Neurosci 30, 1625-1635 (2009). [PubMed: 19811532]

38. Santel S, Baving L, Krauel K, Münte TF \& Rotte M Hunger and satiety in anorexia nervosa: fMRI during cognitive processing of food pictures. Brain Res. 1114, 138-148 (2006). [PubMed: 16919246]

39. Uher R, Treasure J, Heining M, Brammer MJ \& Campbell IC Cerebral processing of foodrelated stimuli: Effects of fasting and gender. Behav. Brain Res 169, 111-119 (2006). [PubMed: 16445991]

40. Fraser S \& Nettle D Hunger affects social decisions in a multi-round Public Goods Game but not a single-shot Ultimatum Game. Adapt. Human Behav. Physiol 6, 334-355 (2020).

41. Häusser JA et al. Acute hunger does not always undermine prosociality. Nat. Commun 10, 4733 (2019). [PubMed: 31628302]

42. Schipper L, Harvey L, van der Beek EM \& van Dijk G Home alone: a systematic review and meta-analysis on the effects of individual housing on body weight, food intake and visceral fat mass in rodents. Obes. Rev 19, 614-637 (2018). [PubMed: 29334694]

43. Matthews GA \& Tye KM Neural mechanisms of social homeostasis. Ann. N.Y. Acad. Sci 10.1111/ nyas.14016 (2019).

44. Liu J et al. Impaired adult myelination in the prefrontal cortex of socially isolated mice. Nat. Neurosci 15, 1621-1623 (2012). [PubMed: 23143512]

45. Sayette MA et al. The measurement of drug craving. Addiction 95, S189-S210 (2000). [PubMed: 11002914]

46. Hawkley LC \& Cacioppo JT Loneliness matters: a theoretical and empirical review of consequences and mechanisms. Ann. Behav. Med 40, 218-227 (2010). [PubMed: 20652462]

47. Dubois J \& Adolphs R Building a science of individual differences from fMRI. Trends Cogn. Sci 20, 425-443 (2016). [PubMed: 27138646]

48. Kohls G, Chevallier C, Troiani V \& Schultz RT Social 'wanting' dysfunction in autism: neurobiological underpinnings and treatment implications. J. Neurodev. Disord 4, 1-20 (2012). [PubMed: 22958445]

49. Nowland R, Necka EA \& Cacioppo JT Loneliness and social internet use: pathways to reconnection in a digital world? Perspect. Psychol. Sci 13, 70-87 (2018). [PubMed: 28937910]

50. Orben A, Dienlin T \& Przybylski AK Social media's enduring effect on adolescent life satisfaction. Proc. Natl Acad. Sci. USA 116, 10226-10228 (2019). [PubMed: 31061122]

51. Faul F, Erdfelder E, Lang AG \& Buchner A G*Power 3: a flexible statistical power analysis program for the social, behavioral, and biomedical sciences. Behav. Res. Methods 39, 175-191 (2007). [PubMed: 17695343]

52. Carter BL \& Tiffany ST Meta-analysis of cue-reactivity in addiction research. Addiction 94, 327340 (1999). [PubMed: 10605857] 
53. Stice E, Burger K \& Yokum S Caloric deprivation increases responsivity of attention and reward brain regions to intake, anticipated intake, and images of palatable foods. Neuroimage 67, 322-330 (2013). [PubMed: 23201365]

54. Stevenson RJ, Francis HM, Attuquayefio T \& Ockert C Explicit wanting and liking for palatable snacks are differentially affected by change in physiological state, and differentially related to salivation and hunger. Physiol. Behav 182, 101-106 (2017). [PubMed: 29030248]

55. Hill RA \& Dunbar RIM Social network size in humans. Hum. Nat 14, 53-72 (2003). [PubMed: 26189988]

56. Lewis PA, Rezaie R, Brown R, Roberts N \& Dunbar RIM Ventromedial prefrontal volume predicts understanding of others and social network size. Neuroimage 57, 1624-1629 (2011). [PubMed: 21616156]

57. Norbeck JS, Lindsey AM \& Carrieri VL The development of an instrument to measure social support. Nurs. Res 30, 264-269 (1981). [PubMed: 7027185]

58. Von Der Heide R, Vyas G \& Olson IR The social network-network: size is predicted by brain structure and function in the amygdala and paralimbic regions. Soc. Cogn. Affect. Neurosci 9, 1962-1972 (2014). [PubMed: 24493846]

59. Duzel E et al. Functional imaging of the human dopaminergic midbrain. Trends Neurosci. 32, 321-328 (2009). [PubMed: 19446348]

60. Pauli WM, Nili AN \& Tyszka JM A high-resolution probabilistic in vivo atlas of human subcortical brain nuclei. Sci. Data 5, 180063 (2018). [PubMed: 29664465]

61. Triantafyllou $\mathrm{C}$ et al. Comparison of physiological noise at $1.5 \mathrm{~T}, 3 \mathrm{~T}$ and $7 \mathrm{~T}$ and optimization of fMRI acquisition parameters. Neuroimage 26, 243-250 (2005). [PubMed: 15862224]

62. Krebs RM, Heipertz D, Schuetze H \& Duzel E Novelty increases the mesolimbic functional connectivity of the substantia nigra/ventral tegmental area (SN/VTA) during reward anticipation: evidence from high-resolution fMRI. Neuroimage 58, 647-655 (2011). [PubMed: 21723396]

63. Bunzeck N \& Duzel E Absolute coding of stimulus novelty in the human substantia nigra/VTA. Neuron 51, 369-379 (2006). [PubMed: 16880131]

64. Schultz W Neural coding of basic reward terms of animal learning theory, game theory, microeconomics and behavioural ecology. Curr. Opin. Neurobiol 14, 139-147 (2004). [PubMed: 15082317]

65. Day JJ, Roitman MF, Wightman RM \& Carelli RM Associative learning mediates dynamic shifts in dopamine signaling in the nucleus accumbens. Nat. Neurosci 10, 1020-1028 (2007). [PubMed: 17603481]

66. Overton P \& Clark D Burst firing in midbrain dopaminergic neurons. Brain Res. Rev 25, 312-334 (1997). [PubMed: 9495561]

67. Schultz W Getting formal with dopamine and reward. Neuron 36, 241-263 (2002). [PubMed: 12383780]

68. Gorgolewski K et al. Nipype: a flexible, lightweight and extensible neuroimaging data processing framework in python. Front. Neuroinform 5, 13 (2011). [PubMed: 21897815]

69. Esteban O, Markiewicz CJ \& Blair RW et al. fMRIPrep: a robust preprocessing pipeline for functional MRI. Nat. Methods 16, 111-116 (2019). [PubMed: 30532080]

70. Tustison NJ et al. Large-scale evaluation of ANTs and FreeSurfer cortical thickness measurements. Neuroimage 99, 166-179 (2014). [PubMed: 24879923]

71. Dale AM, Fischl B \& Sereno MI Cortical surface-based analysis. I. Segmentation and surface reconstruction. Neuroimage 9, 179-194 (1999). [PubMed: 9931268]

72. Zhang Y, Brady M \& Smith S Segmentation of brain MR images through a hidden Markov random field model and the expectation-maximization algorithm. IEEE Trans. Med. Imaging 20, 45-57 (2001). [PubMed: 11293691]

73. Jezzard P \& Balaban RS Correction for geometric distortion in echo planar images from B0 field variations. Magn. Reson. Med 34, 65-73 (1995). [PubMed: 7674900]

74. Abraham A et al. Machine learning for neuroimaging with scikit-learn. Front. Neuroinform 8,14 (2014). [PubMed: 24600388] 
75. Jenkinson M, Bannister P, Brady M \& Smith S Improved optimization for the robust and accurate linear registration and motion correction of brain images. Neuroimage 17, 825-841 (2002). [PubMed: 12377157]

76. Behzadi Y, Restom K, Liau J \& Liu TT A component based noise correction method (CompCor) for BOLD and perfusion based fMRI. Neuroimage 37, 90-101 (2007). [PubMed: 17560126]

77. Greve DN \& Fischl B Accurate and robust brain image alignment using boundary-based registration. Neuroimage 48, 63-72 (2009). [PubMed: 19573611]

78. Smith SM \& Brady JM SUSAN—a new approach to low level image processing. Int. J. Comput. Vis 23, 45-78 (1997).

79. Esteban $\mathrm{O}$ et al. MRIQC: advancing the automatic prediction of image quality in MRI from unseen sites. PLoS ONE 12, e0184661 (2017). [PubMed: 28945803]

80. Vedhara K, Hyde J, Gilchrist ID, Tytherleigh M \& Plummer S Acute stress, memory, attention and cortisol. Psychoneuroendocrinology 25, 535-549 (2000). [PubMed: 10840167]

81. Jenkinson M, Beckmann CF, Behrens TE, Woolrich MW \& Smith SM Fsl Neuroimage 62, 782790 (2012). [PubMed: 21979382]

82. Chowdhury R, Lambert C, Dolan RJ \& Düzel E Parcellation of the human substantia nigra based on anatomical connectivity to the striatum. Neuroimage 81, 191-198 (2013). [PubMed: 23684858]

83. Haber SN, Fudge JL \& McFarland NR Striatonigrostriatal pathways in primates form an ascending spiral from the shell to the dorsolateral striatum. J. Neurosci 20, 2369-2382 (2000). [PubMed: 10704511]

84. Noori HR, Cosa Linan A \& Spanagel R Largely overlapping neuronal substrates of reactivity to drug, gambling, food and sexual cues: a comprehensive meta-analysis. Eur. Neuropsychopharmacol 26, 1419-1430 (2016). [PubMed: 27397863]

85. Tang DW, Fellows LK, Small DM \& Dagher A Food and drug cues activate similar brain regions: a meta-analysis of functional MRI studies. Physiol. Behav 106, 317-324 (2012). [PubMed: 22450260]

86. Kuhn S \& Gallinat J Common biology of craving across legal and illegal drugs-a quantitative meta-analysis of cue-reactivity brain response. Eur. J. Neurosci 33, 1318-1326 (2011). [PubMed: 21261758]

87. Barr DJ, Levy R, Scheepers C \& Tily H J. Random effects structure for confirmatory hypothesis testing: keep it maximal. J. Memory Lang 68, 255-278 (2013).

88. Hausman JA \& Wise DA Social experimentation, truncated distributions, and efficient estimation. Econometrica 45, 919-938 (1977).

89. Im J, Ahn E, Beck N, Kim JK \& Park T Correlation estimation with singly truncated bivariate data. Stat. Med 36, 1977-1988 (2017). [PubMed: 28239899]

90. Hanke M et al. PyMVPA: a python toolbox for multivariate pattern analysis of fMRI data. Neuroinformatics 7, 37-53 (2009). [PubMed: 19184561]

91. Hendriks MHA, Daniels N, Pegado F \& Op de Beeck HP The effect of spatial smoothing on representational similarity in a simple motor paradigm. Front. Neurol 8, 222 (2017). [PubMed: 28611726]

92. Kamitani Y \& Sawahata Y Spatial smoothing hurts localization but not information: pitfalls for brain mappers. Neuroimage 49, 1949-1952 (2010). [PubMed: 19559797]

93. Op de Beeck HP Against hyperacuity in brain reading: spatial smoothing does not hurt multivariate fMRI analyses? Neuroimage 49, 1943-1948 (2010). [PubMed: 19285144]

94. Stelzer J, Chen Y \& Turner R Statistical inference and multiple testing correction in classificationbased multi-voxel pattern analysis (MVPA): random permutations and cluster size control. Neuroimage 65, 69-82 (2013). [PubMed: 23041526]

95. Allefeld C, Görgen K \& Haynes J-D Valid population inference for information-based imaging: from the second-level t-test to prevalence inference. Neuroimage 141, 378-392 (2016). [PubMed: 27450073]

96. Friston KJ, Holmes A, Poline JB, Price CJ \& Frith CD Detecting activations in PET and fMRI: levels of inference and power. Neuroimage 4, 223-235 (1996). [PubMed: 9345513] 
97. Eklund A, Knutsson H \& Nichols TE Cluster failure revisited: impact of first level design and physiological noise on cluster false positive rates. Hum. Brain Mapp 40, 2017-2032 (2019). [PubMed: 30318709]

98. Eklund A, Nichols TE \& Knutsson H Cluster failure: why fMRI inferences for spatial extent have inflated false-positive rates. Proc. Natl Acad. Sci. USA 113, 7900-7905 (2016). [PubMed: 27357684] 
(1) Screening Social connectedness
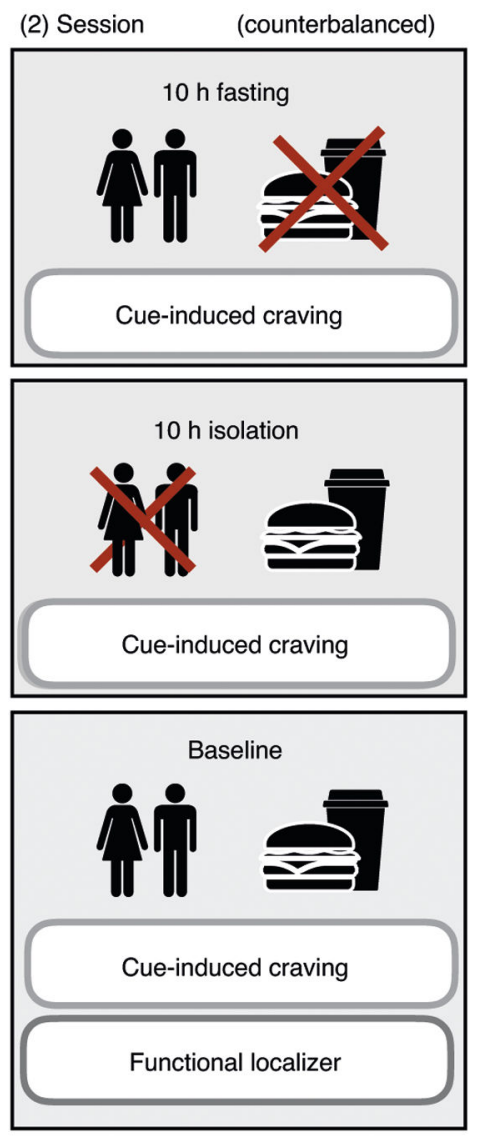

(3) MRI at $7 \mathrm{pm}$ after each session Anatomical and functional scans

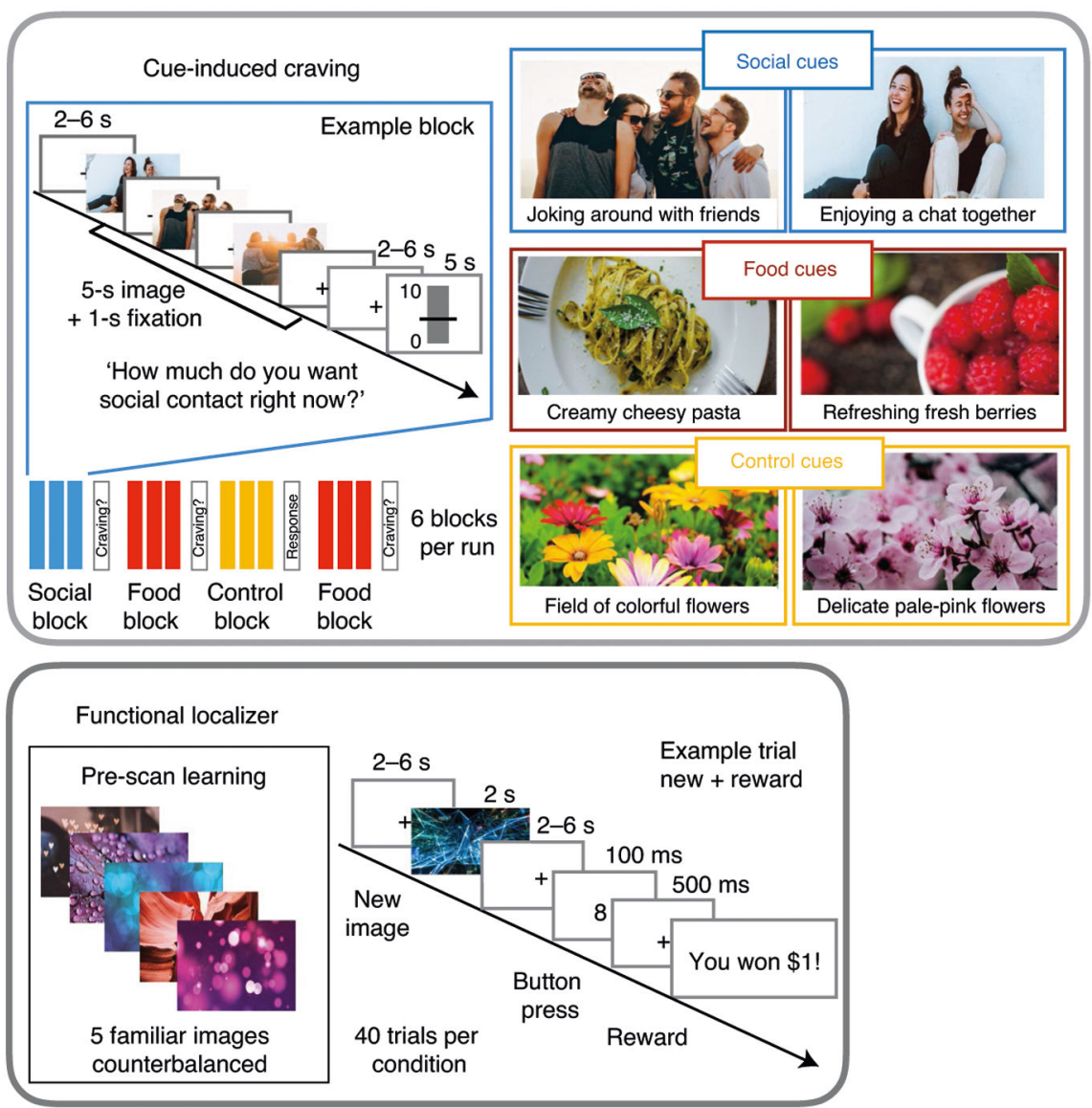

Fig. 1 . Overview of the experimental procedures.

First, individuals underwent screening for their social connectedness (measured by social network size and self-reported loneliness; see Methods for details). each participant ( $n$ $=40$ ) then underwent three experimental sessions: fasting, baseline and isolation (the order of sessions was counterbalanced across participants) and subsequently an MR scan with the CIC task. On the baseline day, participants also underwent a functional localizer task. In the CIC task, participants saw cues for social contact, food and control cues depicting flowers. After each block of cues (showing three images), participants rated their self-reported social craving (after social blocks), food craving (after food blocks) and how much they liked the flower pictures (after control blocks). In the functional localizer task, participants memorized a set of five images before the scan (four different sets of images were counterbalanced across participants). Immediately before the localizer task, participants were shown the memorized pictures again. During the task, participants saw either one of the memorized pictures or a new picture indicating whether or not they would be able to win money. 

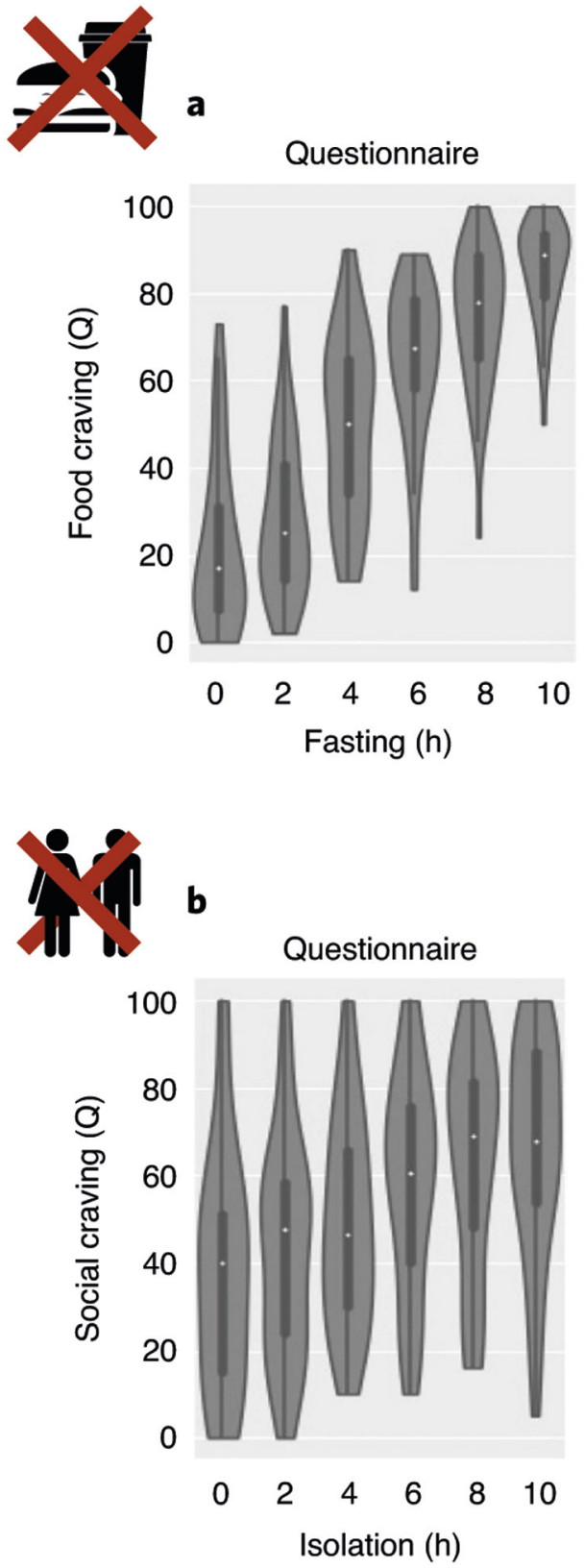

Fasting

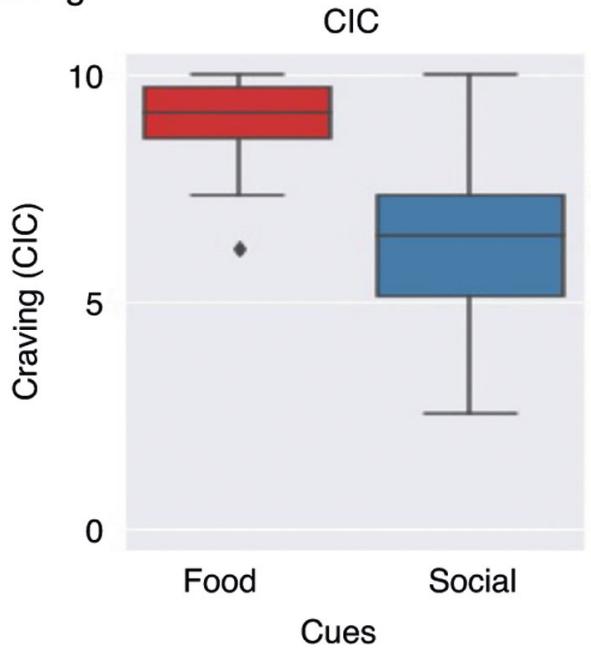

Isolation

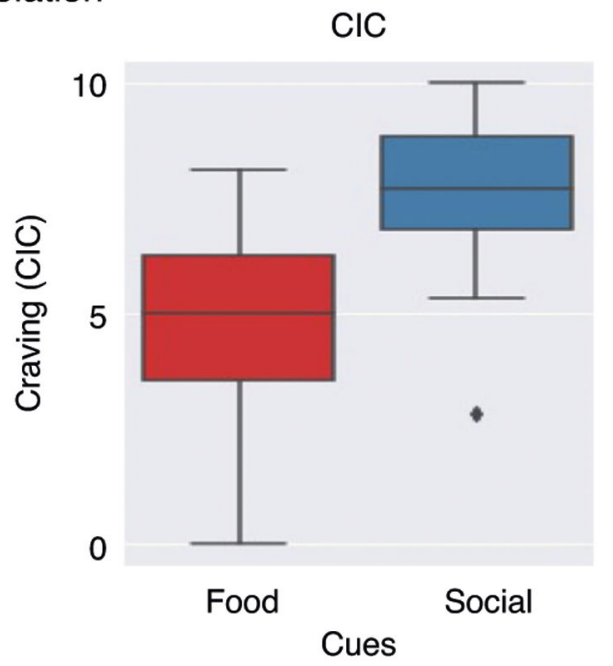

Fig. 2 |. Behavioral results.

a, Changes in self-reported food craving over time during fasting (left; $n=37$ ) and compared with ratings of social craving during the CIC task (right; $n=40$ ). $\mathbf{b}$, Changes in self-reported social craving over time during isolation (left; $n=40$ ) and in comparison to ratings of food craving during the CIC task (right; $n=40$ ). The boxplots in $\mathbf{a}$ and $\mathbf{b}$ indicate the median (dark center line), the interquartile range (IQR; box) and the $1.5 \times \mathrm{IQR}$ minima and maxima (whiskers). Data points outside the whiskers are shown as individual data points. 
a Anatomical SN/NTA
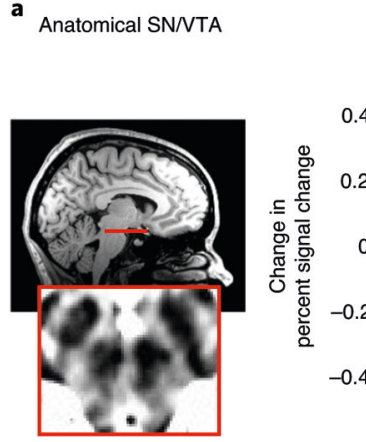

Food $>$ control

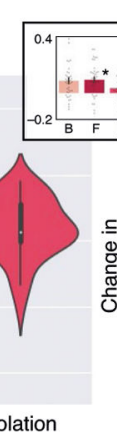

Fasting

Isolation

b

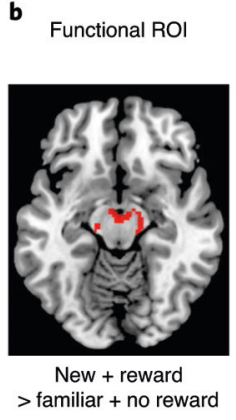

Social > control

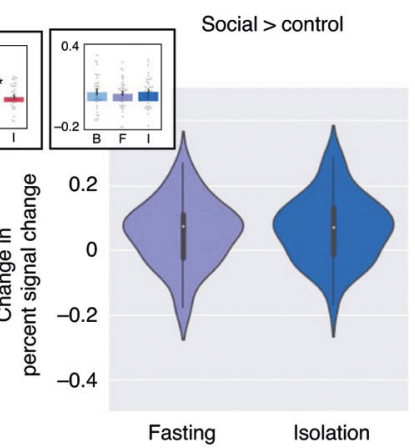

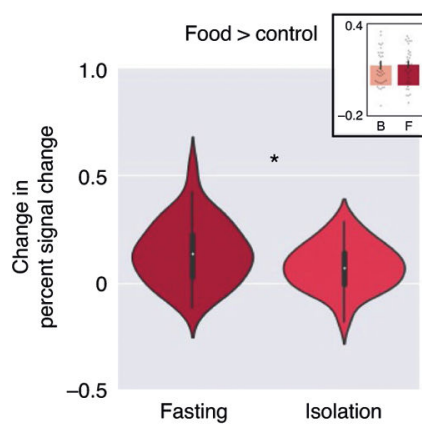

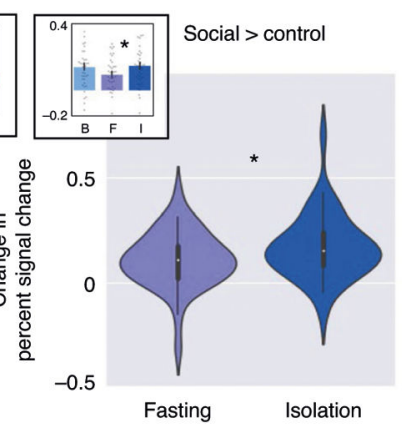

c

Fasting
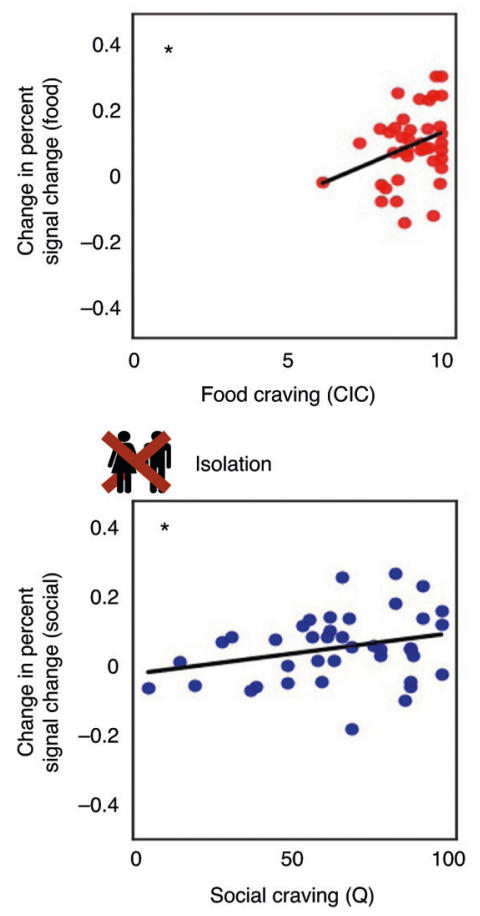

Fig. 3 |. Univariate activity in response to food fasting and social isolation.

a-c, Data $(n=40)$ shown for the anatomical ROI (a), the functional ROI (b) and correlations with self-reported craving (c). The violin plots depict the difference (in percentage signal change) in response to food cues (contrast: food > flowers) and social cues (contrast: social $>$ flowers) after fasting and isolation. The violin plots illustrate the distribution of the data, the white dots indicate the median, the bold dark-gay vertical line the IQR and the thin gray lines the $1.5 \times$ IQR minima and maxima. The insert barplots depict the contrast values for all three sessions: baseline (B), fasting (F) and isolation (I), showing the mean for each session. The error bars indicate the s.e.m. a, Responses in the anatomical SN/VTA were higher for food after fasting compared with isolation. $\mathbf{b}$, Responses in the midbrain functional ROI were higher for food after fasting (compared with isolation) and for social cues after isolation (compared with fasting). c, In the anatomical SN/VTA, responses to food cues were correlated with craving reported in the CIC task, after fasting (top) and responses to social cues were correlated with craving reported on the final questionnaire, after isolation (bottom). 
Training data: fasting per participant

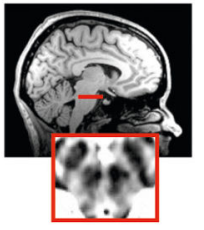

Anatomical SN/VTA

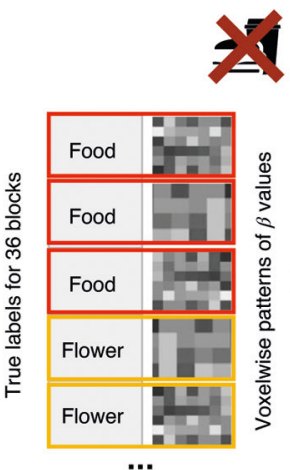

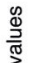

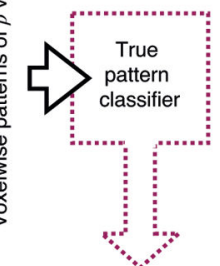

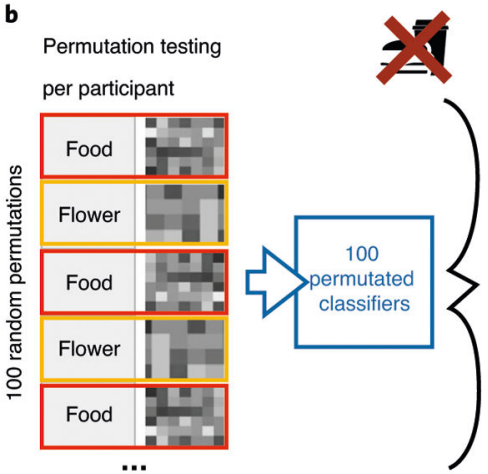

Create null samples:

Randomly sample

one permutated

accuracy value

per participant and

average across

participants

Repeat 10,000 times

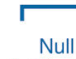
distributions

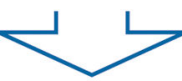

c Testing data: per participant, classify patterns of $\beta$ values in SN/VTA; average accuracy across participants
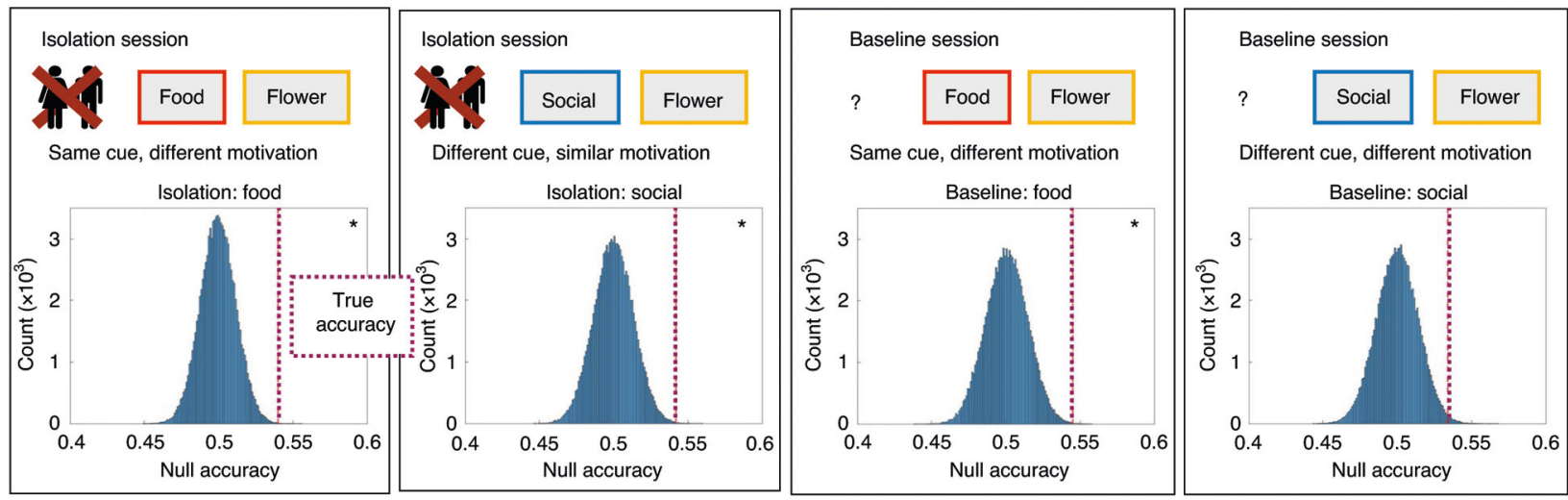

d Representational dissimilarity per participant, average patterns of $\beta$ values in SN/NTA across sessions

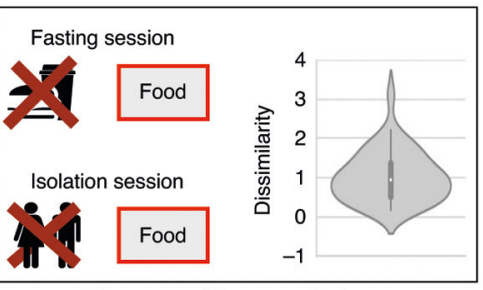

Same cue, different motivation

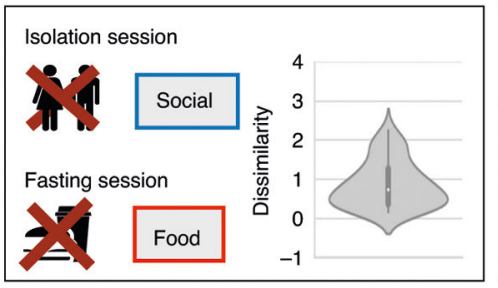

Different cue similar motivation

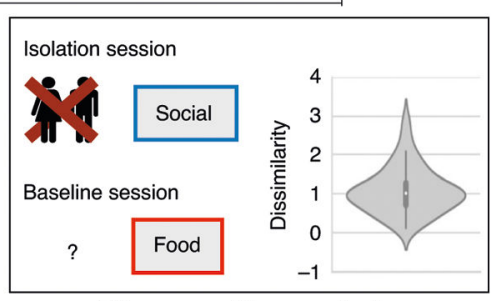

Different cue, different motivation

Fig. 4 . Multivoxel pattern analysis.

a, A linear classifier was trained to distinguish the pattern of activity across voxels in the anatomical SN/VTA of each participant $(n=40)$, in response to food and control cues after fasting. This classifier was then tested on patterns of activity in the same participant in the other two sessions. b, For comparison, we generated null distributions using permutation and bootstrapping procedures: first, we permutated the labels within runs for each participant's training data, and then we tested the permutated classifier on each testing dataset. We created a null distribution by randomly sampling one of the permutated accuracies for each participant and then averaging the accuracy values across subjects. This procedure was repeated $10^{5}$ times to generate the group-level null distributions. c, Null distributions for each testing dataset. The dotted red line shows the true accuracy value (that is, classification accuracy from the real classifier) for each testing dataset. The classifier was generalized to (that is, successfully decoded above chance) food cues (versus control cues) on both 
isolation and baseline days and to social cues (versus control cues) on the isolation day, but not on the baseline day. $\mathbf{d}$, We directly compared the spatial pattern of activity for deprived and nondeprived cues. Note that these results show Fisher-transformed dissimilarity, a measure of representational distance, so lower numbers indicate a more similar spatial pattern. The violin plots illustrate the distribution of the data, the white dots indicate the median, the bold dark-gray vertical line the IQR and the thin gray lines the $1.5 \times \mathrm{IQR}$ minima and maxima. Social cues after isolation were more similar to food cues after fasting than to food cues at baseline. Food cues after fasting were (trending) more similar to social cues after isolation than food cues after isolation. Both $\mathbf{c}$ and $\mathbf{d}$ show that the pattern of activity in SN/VTA is determined not only by the category of visual stimulus, but also by the motivational salience of the category, with craved cues evoking a similar pattern whether what is craved is food or social interaction. 


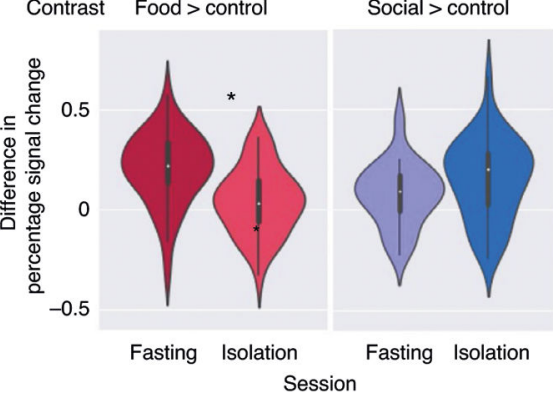

b
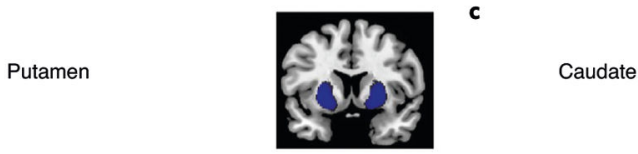

Social $>$ control

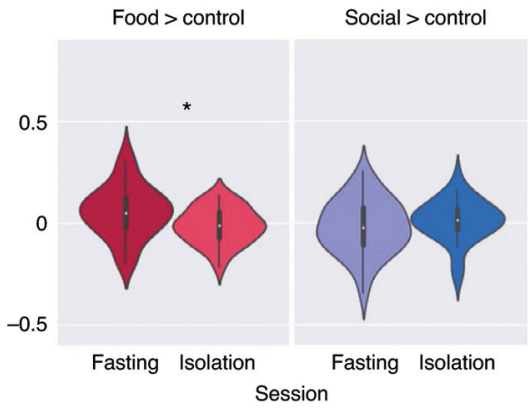

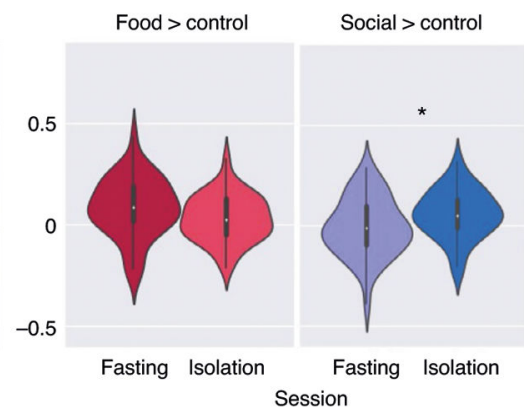

Fig. 5 |. Univariate activity in response to food fasting and social isolation within the striatum. $\mathbf{a}-\mathbf{c}$, The striatum was divided into three subregions: NAcc (a), $\mathrm{Ca}(\mathbf{b})$ and $\mathrm{Pu}(\mathbf{c})$. The violin plots depict the difference (in percentage signal change) in response to food cues (contrast: food > flowers) and social cues (contrast: social > flowers) after fasting and isolation, respectively. The violin plots illustrate the distribution of the data $(n=40)$, the white dots indicate the median, the bold dark-gray vertical line the IQR and the thin gray lines the $1.5 \times$ IQR minima and maxima. 